\title{
Genomic mechanisms of climate adaptation in polyploid bioenergy switchgrass
}

https://doi.org/10.1038/s41586-020-03127-1

Received: 1 July 2020

Accepted: 16 December 2020

Published online: 27 January 2021

Open access

Check for updates

\author{
John T. Lovell ${ }^{1,38 凶}$, Alice H. MacQueen ${ }^{2,38}$, Sujan Mamidii, ${ }^{1,38}$, Jason Bonnette ${ }^{2,38}$, \\ Jerry Jenkins ${ }^{1,38}$, Joseph D. Napier ${ }^{2}$, Avinash Sreedasyam' ', Adam Healey' , Adam Session ${ }^{3,4}$, \\ Shengqiang Shu ${ }^{3}$, Kerrie Barry ${ }^{3}$, Stacy Bonos ${ }^{5}$, LoriBeth Boston', Christopher Daum ${ }^{3}$, \\ Shweta Deshpande ${ }^{3}$, Aren Ewing ${ }^{3}$, Paul P. Grabowski', Taslima Haque ${ }^{2}$, Melanie Harrison ${ }^{6}$, \\ Jiming Jiang ${ }^{7}$, Dave Kudrna ${ }^{8}$, Anna Lipzen ${ }^{3}$, Thomas H. Pendergast IV ${ }^{9,10,11}$, Chris Plott', \\ Peng Qi ${ }^{9}$, Christopher A. Saski ${ }^{12}$, Eugene V. Shakirov ${ }^{2,13}$, David Sims ${ }^{1}$, Manoj Sharma ${ }^{14}$, \\ Rita Sharma ${ }^{15}$, Ada Stewart', Vasanth R. Singan ${ }^{3}$, Yuhong Tang ${ }^{16}$, Sandra Thibivillier ${ }^{17}$, \\ Jenell Webber', Xiaoyu Weng' ${ }^{2}$, Melissa Williams', Guohong Albert $\mathbf{W u}^{3}$, Yuko Yoshinaga ${ }^{3}$, \\ Matthew Zane ${ }^{3}$, Li Zhang ${ }^{2}$, Jiyi Zhang ${ }^{16}$, Kathrine D. Behrman ${ }^{2}$, Arvid R. Boe ${ }^{18}$, Philip A. Fay ${ }^{19}$, \\ Felix B. Fritschi ${ }^{20}$, Julie D. Jastrow ${ }^{21}$, John Lloyd-Reilley ${ }^{22}$, Juan Manuel Martínez-Reyna ${ }^{23}$, \\ Roser Matamala ${ }^{21}$, Robert B. Mitchell ${ }^{24}$, Francis M. Rouquette $\mathrm{Jr}^{25}$, Pamela Ronald ${ }^{26,27}$, \\ Malay Saha ${ }^{16}$, Christian M. Tobias ${ }^{28}$, Michael Udvardi ${ }^{16}$, Rod A. Wing ${ }^{8}$, Yanqi Wu ${ }^{29}$, \\ Laura E. Bartley ${ }^{30,31}$, Michael Casler ${ }^{32,33}$, Katrien M. Devos ${ }^{9,10,11,34}$, David B. Lowry ${ }^{7,35}$, \\ Daniel S. Rokhsar ${ }^{3,4,36,37}$, Jane Grimwood' ${ }^{\text {, Thomas E. Juenger }}{ }^{2 \bowtie}$ \& Jeremy Schmutz ${ }^{1,3 凶}$
}

Long-term climate change and periodic environmental extremes threaten food and fuel security ${ }^{1}$ and global crop productivity ${ }^{2-4}$. Although molecular and adaptive breeding strategies can buffer the effects of climatic stress and improve crop resilience $^{5}$, these approaches require sufficient knowledge of the genes that underlie productivity and adaptation ${ }^{6}-$ knowledge that has been limited to a small number of well-studied model systems. Here we present the assembly and annotation of the large and complex genome of the polyploid bioenergy crop switchgrass (Panicum virgatum). Analysis of biomass and survival among 732 resequenced genotypes, which were grown across 10 common gardens that span $1,800 \mathrm{~km}$ of latitude, jointly revealed extensive genomic evidence of climate adaptation. Climate-gene-biomass associations were abundant but varied considerably among deeply diverged gene pools. Furthermore, we found that gene flow accelerated climate adaptation during the postglacial colonization of northern habitats through introgression of alleles from a pre-adapted northern gene pool. The polyploid nature of switchgrass also enhanced adaptive potential through the fractionation of gene function, as there was an increased level of heritable genetic diversity on the nondominant subgenome. In addition to investigating patterns of climate adaptation, the genome resources and gene-trait associations developed here provide breeders with the necessary tools to increase switchgrass yield for the sustainable production of bioenergy.
Switchgrass (P. virgatum) is both a promising biofuel crop and an important component of the North American tallgrass prairie. Historically, tallgrass prairies were one of the largest temperate biomes on Earth, and they remain important sinks for atmospheric carbon ${ }^{7,8}$. However, most extant natural switchgrass populations are restricted to 'relic' sites, which represent crucial but dwindling genetic resources for the future conservation and breeding of tallgrass prairie.

Biomass production is the principal breeding target for switchgrass as a forage and bioenergy crop ${ }^{9}$ and is a strong proxy for seed production and evolutionary fitness ${ }^{10}$. Since the US Department of Energy named switchgrass a model herbaceous biofuel feedstock, biomass yield trials have demonstrated the economic viability of switchgrass bioenergy production, and cultivars have been bred that substantially out-produce maize and other cellulosic feedstocks ${ }^{11}$. However, individual cultivars tend to be productive across only a narrow climatic niche. Therefore, to maximize gains, switchgrass breeding and biotechnology should focus on developing climate-genotype matches ${ }^{12,13}$ through the identification of the genomic basis of biomass accumulation and climate adaptation in breeding panels. This will bolster future yields $\mathbf{s}^{14}$ and cement switchgrass as an economically and environmentally sustainable bioenergy product.

\section{The tetraploid switchgrass genome}

Although abundant quantitative genetic variation underlies climate-associated stress tolerance and biomass production ${ }^{15,16}$, the 

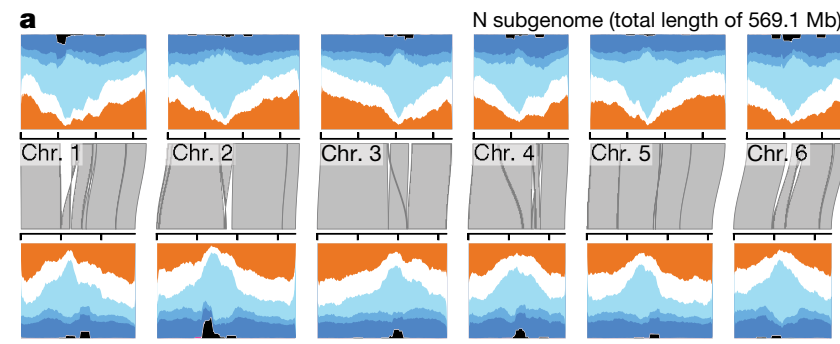

K subgenome (total length of $529.3 \mathrm{Mb}$ )

Fig. 1 | The structure and evolution of the subgenomes of tetraploid switchgrass. a, Grey polygons (representing $n=53$ syntenic blocks) demonstrate nearly complete co-linearity between subgenomes. Gene-rich chromosome arms and highly repetitive pericentromeres are typical of grass

fragmented and incomplete nature of previous switchgrass genome sequences have impeded the discovery of candidate genes and other molecular breeding efforts. The genome of the AP13 switchgrass genotype is large (haploid genome size $=1,129.9$ megabases $(\mathrm{Mb})$ ), repetitive (56.9\% repeats) (Fig. 1a, Extended Data Fig. 1 ) and polyploid. In contrast to some other outcrossing species such as maize (which is represented by the inbred B73 reference genome), AP13 is outbred. Its genome retains a commensurate level of heterozygosity within the range of naturally outcrossing populations (Extended Data Fig. 1). Despite this complexity, our deep PacBio long-read sequencing coupled with deep short-read polishing and bacterial artificial chromosome (BAC) clone validation produced a highly contiguous ' $v 5$ ' AP13 genome assembly (Extended Data Fig. 1; data are available from Phytozome at https:// phytozome-next.jgi.doe.gov). We pruned the resulting large contigs $\left(\mathrm{N}_{50}=5.5 \mathrm{Mb}\right)$ to a single representative haplotype, and then oriented and ordered into chromosome pseudomolecules using the consensus of two high-density genetic maps (Supplementary Data 1). Chromosomes were assigned to subgenomes via genetic distance to Panicum rudgeii $^{17}$ (the sister taxa to the K subgenome of $P$. virgatum), and via de novo repeat clustering. The final assembly contains only $0.4 \%$ gaps, a 75-fold decrease relative to a previous $v 4$ release from 2016 (https:// phytozome-next.jgi.doe.gov/info/Pvirgatum_v4_1). Importantly, the genome assembly was co-linear with three sources of genetic information, despite being assembled independently from all three: the assembly of a close diploid relative (Panicum hallii), the marker order of a pseudo- $\mathrm{F}_{2}$ genetic map and the gene order of the alternative subgenome (Fig. 1a, Extended Data Fig. 1, Supplementary Data 2). These co-linearities demonstrated that we have developed a single haploid assembly and annotation for each subgenome.

Crucially, we were able to distinguish gene and repeat sequences between the two subgenomes. The gene annotation-which is derived from Illumina RNA sequencing $\left(n_{\text {libraries }}=88, n_{\text {conditions }}=18,>3\right.$ billion reads) and PacBio Iso-Seq ( $n_{\text {conditions }}=9,>4.5$ million reads, Supplementary Data 3 )-encompasses 80,278 primary and 49,664 alternative transcripts and is as complete as the genome assembly (BUSCO $=99.4 \%$ ) (Extended Data Fig. 1). We leveraged these annotations to build multiple sequence alignments and time-scaled phylogenetic trees, which date subgenome-progenitor species divergence to about 6.7 million years ago (Ma). Long-terminal repeat sequence analysis of subgenome-specific proliferation of retrotransposons sets an upper bound of the polyploidy event that formed switchgrass at $\leq 4.6 \mathrm{Ma}$ (Fig. 1b), which indicates that tetraploid switchgrass arose during the Pliocene, or the glacial-interglacial cycles of the early Pleistocene epoch.

\section{Climate adaptation drives biomass yield}

Although there are two reproductively isolated ${ }^{18}$ switchgrass cytotypes (tetraploid $(4 \times)$ and octoploid $(8 \times)$ ), tetraploids represent the majority of cultivars ${ }^{19}$ and span a broader geographical range than octoploids ${ }^{20}$.
To investigate the genetic basis of climate adaptation, stress tolerance and biomass production, we therefore developed a diversity panel of 732 exclusively tetraploid genotypes (Supplementary Data 4). We clonally propagated and transplanted this panel in up to 10 common gardens that spanned $1,862 \mathrm{~km}$ of latitude, from southern Texas to South Dakota (USA) $\left(n_{\text {plants }}=5,521\right)$ (Fig. 2a) and resequenced each genotype via deep $($ median $=59 \times)$ coverage $2 \times 150$-bp paired-end PCR-free Illumina libraries. Importantly, resequencing coverage was not biased towards either subgenome (likelihood ratio test $\chi^{2}=1.32$, degrees of freedom $=1$, $P=0.25)$. Our resequencing yielded 33.8 million single-nucleotide polymorphisms (SNPs) (minor allele frequency $\geq 0.5 \%$ ) mapped against the genome. We also de novo-assembled a 252-genotype subset of these deeply resequenced libraries and called presence-absence and structural variants (for example, 100-1,500-bp insertions and deletions) on the resulting contigs. To connect trait and molecular variation with climate, we extracted 46 climate variables ${ }^{21,22}$ from the georeferenced collection location of each genotype and clustered these data into seven groups that explained the majority of climatic variation across the diversity panel (Extended Data Fig. 2).

Climate-associated adaptation in switchgrass has previously been hypothesized to underscore divergence between northern upland and southern lowland ecotypes and is exemplified by divergent leaf and whole-plant morphologies ${ }^{13,23-26}$. In silico classification from morphological data, coupled with ecotype assignments by experts across our diversity panel (Supplementary Data 5), revealed upland $(n=268)$, lowland $(n=99)$ and a third, coastal ecotype $(n=184)$. The coastal ecotype was broadly sympatric with the lowland ecotype but displayed upland leaf characters and lowland plant architecture (Fig. 2a, Extended Data Fig. 2).

We observed strong evidence that adaptive evolution has contributed to ecotype divergence. Whereas winter-kill mortality was rare among northern upland plants (2.4\%), nearly half of all coastal (42.1\%) and lowland (42.8\%) genotypes perished during the winter of 2018-2019 across the 4 northernmost gardens (Fig. 2b). Winter kill was especially severe in the three northwestern plains sites, probably owing to a period of severe cold from late January to early March 2019 (Extended Data Fig. 2). In total, genotypes from the northern $30 \%$ of the panel were $218 \times\left(\right.$ Fisher's test odds ratio $\left.=218.17, P<1 \times 10^{-15}\right)$ more likely to survive the winter of 2018-2019 in the northern 4 sites than the southernmost $30 \%$ of the genotypes.

The latitude gradient across our common gardens also served as the major axis of biomass variation. Among the seven groups of correlated climatic variables, the strongest predictors of biomass variation were always related to temperature (Extended Data Fig. 2). We observed particularly strong signals of extreme 30 -year-minimum temperature as a predictor of biomass in the winter-kill-susceptible lowland and coastal ecotypes (Fig. 2c). For both ecotypes, genotypes collected from sites with colder historical extreme minimum temperatures out-performed genotypes from sites with a milder climate in the northern gardens. 
a

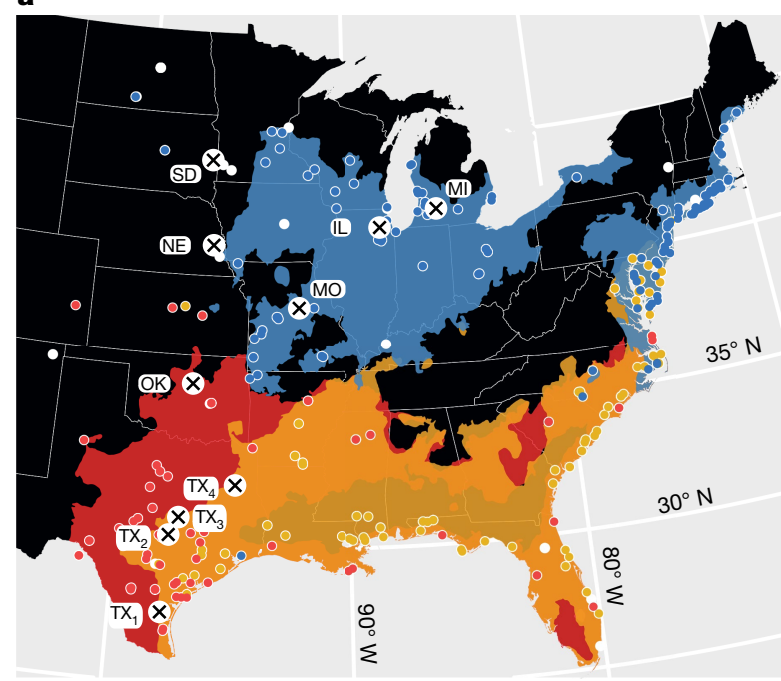

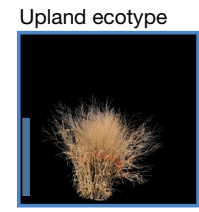

Coastal ecotype

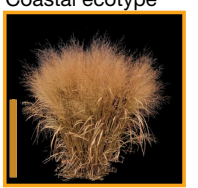

Lowland ecotype

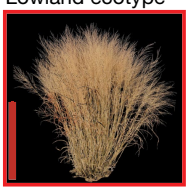

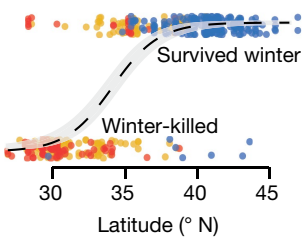

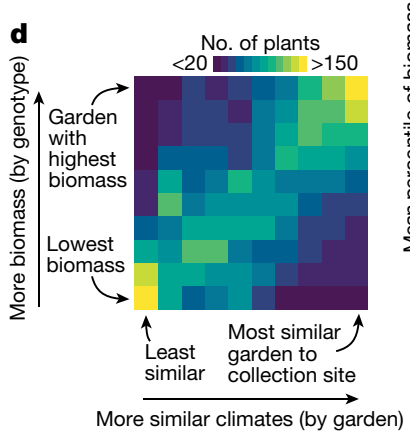

c

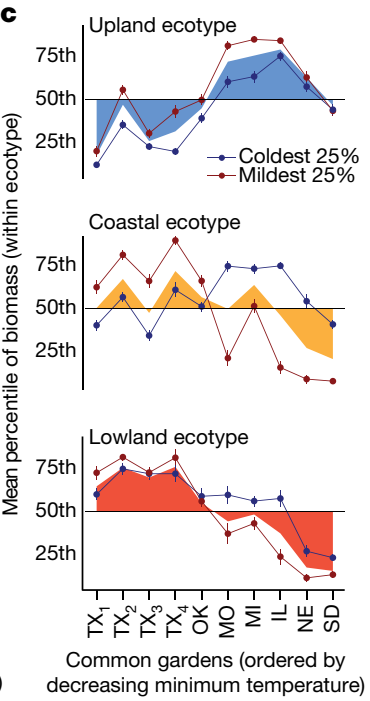

Fig. 2 | Climatic adaptation within and among switchgrass ecotypes. a, Geographical distribution of common gardens $(n=10)$ and plant collection locations ( $n=700$ georeferenced genotypes), and spatial distribution models of each ecotype. The ecotype colour legend accompanies the representative images of each ecotype to the right of the map (images were taken at the end of the 2019 growing season and the background was removed with ImageJ (https://imagej.nih.gov/ij)). White-outlined points (coloured by ecotype, or in white if no ecotype assignment was made) indicate the georeferenced collection sites of the diversity panel. The labelled white circles with black crosses indicate the locations of the 10 experimental gardens. Publicly available cultural and physical geographical information system (GIS) layers were accessed with the rnaturalearthdata R package ${ }^{51}$.Scale bars, $1 \mathrm{~m}$. b, Across the landscape, survival $\left(n_{\text {genotypes }}=367\right)$ and winter kill $(n=184)$ in the northern gardens $(n=3)$ was geographically structured: the latitude of the origin of

However, no climate-of-origin-dependent trade-off was observed in the winter-kill-tolerant upland ecotype. It is possible that a more intensely cold winter than that of 2018-2019 could introduce differential survival in the upland genotypes and produce a trade-off similar to that observed within the two more southern ecotypes. These results add support to our observation that susceptibility to cold temperatures acts both as an agent of natural selection and as a limiter of northern range expansion.

Furthermore, biomass yield for each genotype was generally maximized in the gardens with climates that were most similar to their collection locations (Fig. 2d). As such, local adaptation is manifest not only through survival and stress tolerance, but also through higher biomass accumulation in climates similar to those in which each genotype evolved.

\section{Ecotype convergence among gene pools}

Knowledge of the structure and diversity of gene pools within switchgrass is critical to projecting future gains from molecular breeding and understanding the genetic basis of climate adaptation ${ }^{12,13}$. Several previous population genetic studies of switchgrass assumed that there should be strong correspondence between population genetic structure and the morphological clustering that is used to define ecotypes ${ }^{20,27,28}$. Analysis of our 33.8-million genome-wide SNP database revealed that our diversity panel is strongly subdivided into three major genetic subpopulations that are, in general, geographically distinct (which we refer to as Midwest, Atlantic and Gulf) (total $F_{\mathrm{ST}}=0.27$ ) (Fig. 3a). The clustering of presence-absence and structural variants largely recapitulates SNP-based subpopulation structure (Extended Data Fig. 3), providing consistent evidence of subpopulation differentiation that may include large-effect mutations at several molecular scales. collection site was predictive of survival. A logistic regression prediction ( \pm s.e.) accompanies binary survival along the latitude predictor.c, Imputed survival-corrected biomass was converted to percentiles for each ecotype ( 0 = lowest biomass, $100=$ highest $)$ and mean percentiles were plotted overall (coloured polygons, $n=447)$ for each ecotype $\left(n_{\text {upland }}=211, n_{\text {coastal }}=144\right.$, $n_{\text {lowland }}=92$ ) and garden. The biomass percentiles (mean \pm s.e.m.) for the $25 \%$ of genotypes from sites with the coldest extreme 30 -year coldest minimum temperature (blue lines and points) $\left(n_{\text {upland }}=52, n_{\text {coastal }}=35, n_{\text {lowland }}=22\right)$ and the mildest $25 \%$ (red lines and points) $\left(n_{\text {upland }}=53, n_{\text {coastal }}=36, n_{\text {lowland }}=23\right)$ demonstrate that climate of origin affects biomass within ecotypes and across gardens. d, A heat map of the rank of climate similarity ( $x$ axis) and imputed biomass ( $y$ axis) demonstrates that the majority of 571 genotypes achieve their highest biomass at common gardens that were climatically similar to their source habitat.

Population genetic structure was discordant with variation in morphological ecotype, which segregated strongly within genetic subpopulations. Plants with upland ecotype traits were present in both the Atlantic (37\%) and Midwest (63\%) gene pools. Similarly, 54\% and $46 \%$ of coastal ecotype accessions were assigned to Atlantic and Gulf subpopulations, respectively (Fig. 3a). All plants with lowland morphology were clustered within the Gulf subpopulation. However, these Gulf lowland plants had approximately equal proportions of individuals that survived and perished during the northern winter (Fig. 2c). Thus, important genetic diversity for breeding was present within genetic subpopulations-a pattern that was validated through realized genetic gains of biomass and winter survival within several switchgrass breeding populations ${ }^{29,30}$.

Despite ecotypic convergence among subpopulations, coalescent simulations dated the divergence of the subpopulations to the mid-Pleistocene epoch (>358,000 generations (0.7-1.4 Ma, assuming a 2-4 year generation time)) (Extended Data Fig. 3). Thus, extant switchgrass gene pools have been diverging for nearly half of the evolutionary history of polyploid switchgrass. In contrast to the deep sequence divergence among subpopulations, we observed very little molecular genetic differentiation between upland and coastal ecotypes within the Atlantic subpopulation $\left(F_{\mathrm{ST}}=0.03\right)$, or between lowland and coastal ecotypes within the Gulf subpopulation $\left(F_{\mathrm{ST}}=0.03\right)$ (Extended Data Fig. 3$)$.

Admixture appears to be common between the Gulf and Atlantic subpopulations; comparisons of plants with coastal ecotype traits from both of these subpopulations were molecularly more similar $\left(F_{\mathrm{ST}}=0.19\right)$ than for noncoastal Gulf and Atlantic plants $\left(F_{\mathrm{ST}}=0.24\right)$. By contrast, the plants with upland morphologies in the Midwest and Atlantic subpopulations were no more similar than other plants from those subpopulations $\left(F_{\mathrm{ST}}\right.$ for both $\left.=0.30\right)$. This convergence of upland morphologies in two highly differentiated genetic subpopulations 


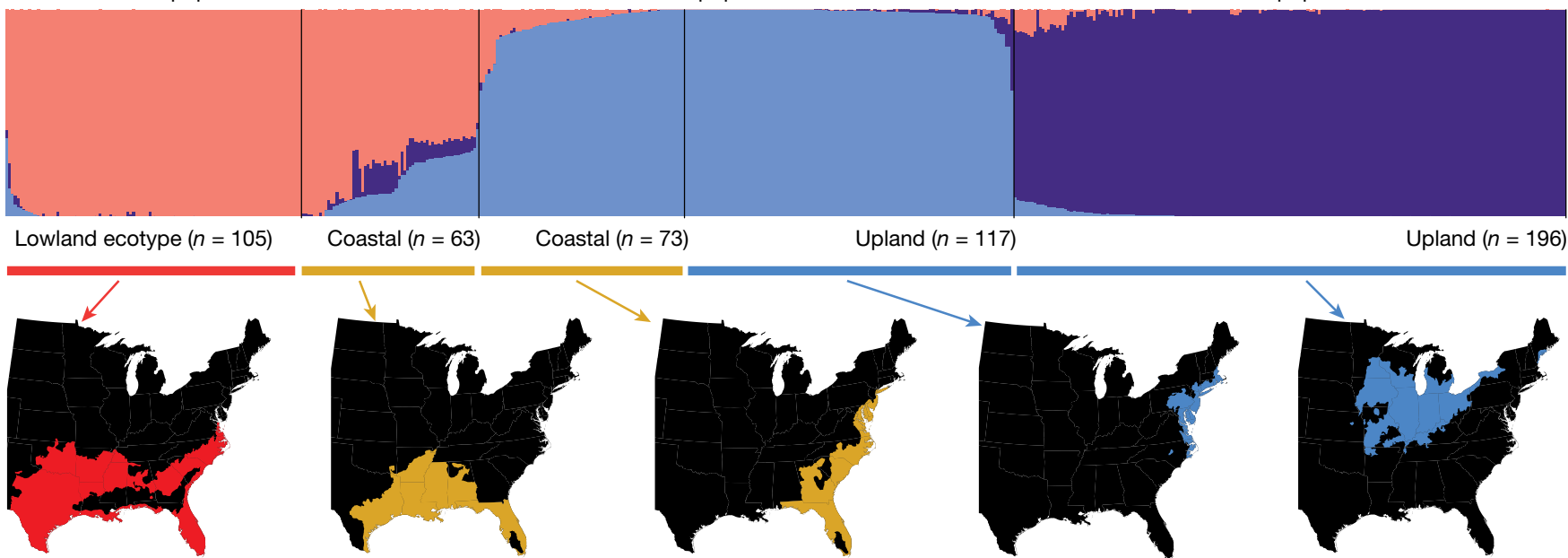

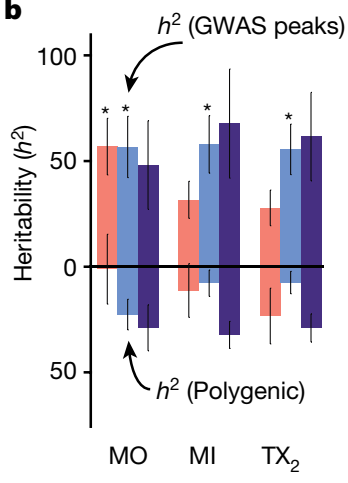

Gardens (biomass)

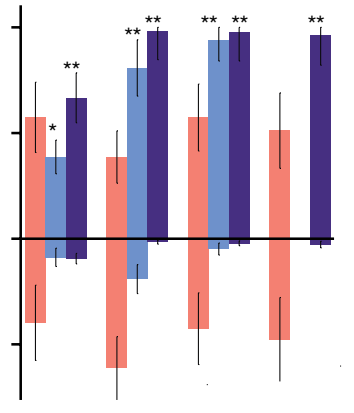

ahm bio16 bio17 bio2

Climate (precipitation)

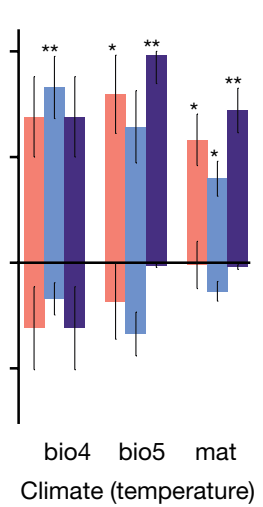

Climate mash

(between subpopulations) Fitness mash

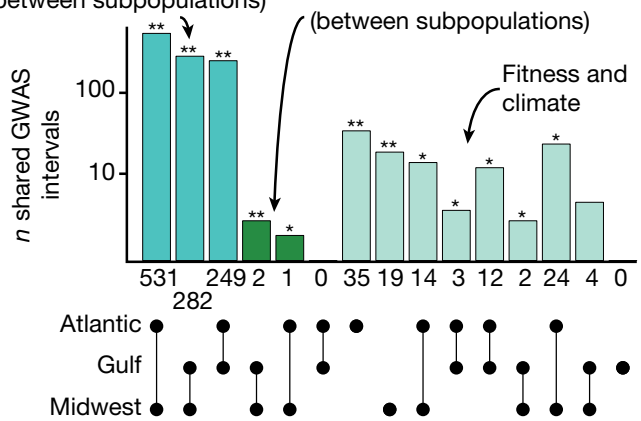

Fig. 3 | Population and quantitative genomics of climate-associated adaptation. a, Admixture proportions among three gene pools (coloured by subpopulation) and three ecotypes (labelled below), calculated using eigenvector decomposition of the identity-by-descent matrix. The corresponding geographical distribution of each ecotype is presented below the bar plot (coloured by the ecotype distributions from Fig.1a). Publicly available cultural and physical GIS layers were accessed with the rnaturalearthdata R package ${ }^{51}$. b. Post hoc tests of SNP-heritability (mean $h^{2} \pm$ s.e.m.) attributable to polygenic background (below the black horizontal lines) and significant multivariate adaptive shrinkage GWAS hits (above the black horizontal lines) are presented for the three main sites (biomass) and for precipitation- and temperature-related climate variables, and coloured by subpopulations (following a). Extended Data Fig. 2c provides descriptions of the climate variables (ahm, bio2, bio4, bio5, bio16, bio17 and mat). Statistical significance of higher heritability for GWAS hits relative to polygenic inheritance is indicated for two-sided $Z$-score $P$ values; ${ }^{* *} P<0.001,{ }^{*} P<0.05$. c, There are large and significant overlaps in climate-associated multivariate adaptive shrinkage (mash) intervals between subpopulations, and smaller but significant overlaps between fitness and climate hits in the Atlantic and Midwest subpopulations. Two-sided Fisher's test $P$ value significance, following $\mathbf{b}$. could be the result of independent genetic origins of the upland ecotype or rare but evolutionarily important ${ }^{31}$ admixture events. We evaluate these hypotheses below.

\section{Genetic targets for yield improvement}

To detect the genetic basis of climate adaptation and fitness within the diversity panel, we conducted multivariate adaptive shrinkage ${ }^{32}$ on genome-wide association mapping (GWAS) results within and across genetic subpopulations. Multivariate adaptive shrinkage shares GWAS peak effect size and direction between univariate tests to improve power to detect significant, shared results. Multivariate adaptive shrinkage results were determined for both fitness GWAS (which mapped winter survival and biomass in the three largest common gardens (MI, MO and $\mathrm{TX}_{2}$ )), and climate GWAS (which detected associations between SNP variation and the climate of origin (seven representative climate variables)). To make direct comparisons among subpopulations (which have different segregating SNPs), we summarized the 12,239 significant linkage-disequilibrium block 'peaks' of multivariate adaptive shrinkage $\left(\log _{10} \text {-transformed Bayes factor }>2\right)^{33}$ into 10,09020 -kb regions ( $20 \mathrm{~kb}$ represents the inflection point at which linkage disequilibrium decay flattens) (Extended Data Fig. 3$)$ for climate $\left(n_{\text {regions }}=9,856\right)$ and fitness $\left(n_{\text {regions }}=332\right)$ GWAS (Supplementary Data 6). A weighted list of candidate genes-including putative SNP effects, the existence of presence-absence or structural variants, gene co-expression and physical proximity to the GWAS peaks-can be found in Supplementary Data 7.

GWAS peaks explained the majority of heritable phenotypic and climatic variation (SNP-heritability) both across and within gene pools (Fig. 3b). SNP-heritability of fitness $\left(h^{2}=51.5 \pm 15.4 \%\right.$ (mean \pm s.e.m.) and climate-associated peaks $\left(h^{2}=70.5 \pm 14.0 \%\right)$ collectively explained over threefold-more variation than the polygenic background (fitness $=19.5 \pm 9.1 \%$, climate $=18.2 \pm 9.5 \%)($ Extended Data Table 1$)$. The high heritability of these climate and biomass associations indicated that relatedness at a small subset of all variants out-predicted overall 


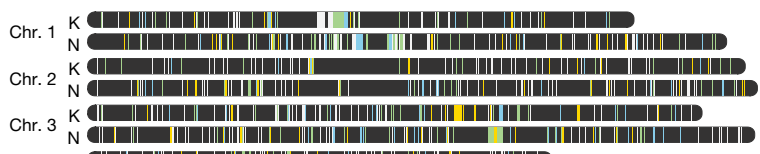

Chr. 4 K A

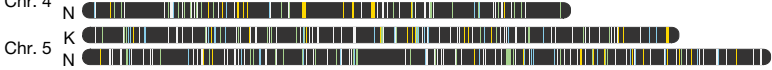

Chr. $6 \mathrm{~K}$ 每

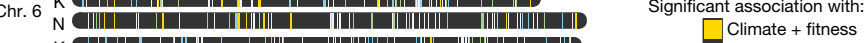

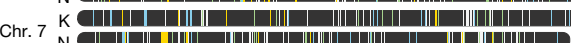

Chr. $7 \underset{\mathrm{N} C \mathrm{~K}}{\mathrm{~K}}$

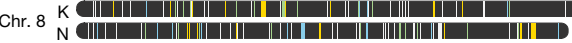

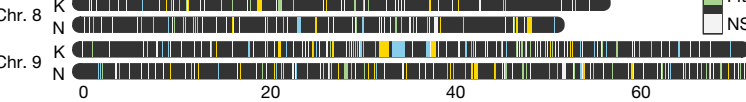

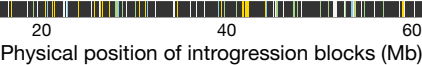

b

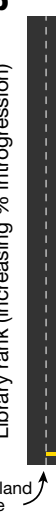

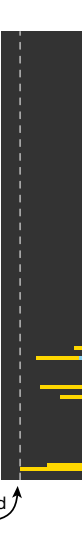

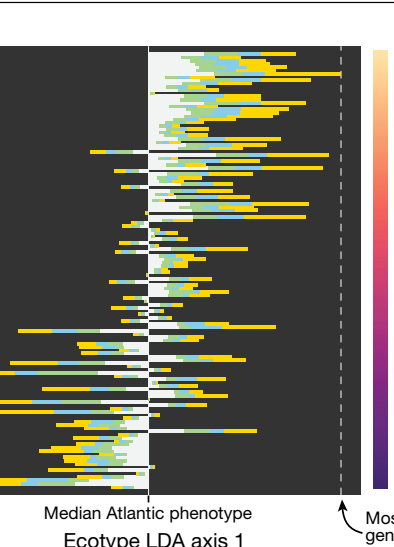

c

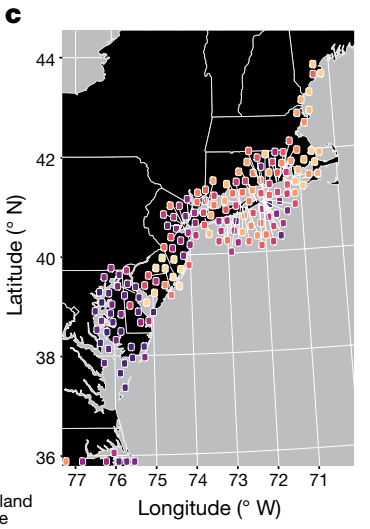

Fig. 4 | Mapping the location and effect of Midwest introgressions in the Atlantic subpopulation. a, Positions of all high-frequency (present in $>10$ genotypes, $n=1,640$ ) introgressions from Midwest into the Atlantic subpopulation are coloured by significance in the two redundancy analyses for climate (blue, $n=234$ ), biomass and survival (green, $n=329$ ) or 'climate-fitness overlap', which are significant in both (gold, $n=245$ ). NS, not significant. b, Introgressions are strongly associated with a more upland phenotype among 135 genotypes. For each genotype, the position along the first discriminant axis between ecotypes (Extended Data Fig. 2) is scaled relative to the median Atlantic ecotype value, then plotted and coloured by the proportion of introgressed sequence in each significance bin from a.c. The introgression ranks from $\mathbf{b}$ were converted to a purple-orange colour scale (right of b) and georeferenced positions of collection sites for each library are plotted for the northern Atlantic seaboard of the USA. Publicly available cultural and physical GIS layers were accessed with the rnaturalearthdata $R$ package $e^{51}$. relatedness and provides breeders with genetic diversity to target for switchgrass improvement in local environments.

Loci that are associated with both fitness and climate of origin are probably involved in local adaptation ${ }^{34}$, and are strong targets for the breeding of locally adapted cultivars. Overall, we observed nearly $2 \times$ more overlap of $20-\mathrm{kb}$ regions associated with both climate and fitness than expected by chance (Fisher's test odds ratio $=1.92, P<1 \times 10^{-6}$ ). This overlap was especially strong within the two northern subpopulations (Midwest, odds $=11.5 \times$ and $P<1 \times 10^{-15}$; Atlantic, odds $=17.8 \times$ and $P<1 \times 10^{-15}$ ) (Fig. 3c), where we expected to see the strongest effect of selection on survival during cold winters.

Many regions of climate and fitness overlap were polymorphic only within a single genetic subpopulation, which highlights several, possibly independent, genetic paths to climate adaptation in switchgrass. However, $9.5 \%$ (940) of the 20 -kb climate intervals were polymorphic in several genetic subpopulations. Given the substantial evidence of admixture between the Gulf and Atlantic subpopulations (Fig. 3a), we expected that contemporary gene flow would be the major contributor to shared polymorphisms. Contrary to this hypothesis, the majority (511 regions) of all multi-subpopulation GWAS intervals were shared between the two most genetically distinct gene pools (Atlantic and Midwest). Given the deep divergence time between these subpopulations, rare or ancient gene flow ${ }^{35}$ may have created these shared adaptive polymorphic regions.

\section{Evolutionary convergence via introgression}

To explicitly address how introgressions may have shaped the distribution of climate-SNP associations, we investigated physically contiguous regions of admixture across the genome using a hidden Markov mode $^{36}$. Introgressions between subpopulations represented $2.98 \%$ of the content of our resequenced genomes (Fig. $4 \mathrm{a}$ ), but were $>1.5 \times$ more likely to contain shared GWAS intervals across subpopulations than expected by chance (Fisher's test odds ratio $=1.55, P<1 \times 10^{-8}$ ), indicating that adaptive introgressions underlie at least a portion of heritable variants shared among subpopulations.

Of particular interest were a suite of introgressions from the Midwest to the Atlantic subpopulation that dated to about 8,700 generations before present (17-34 thousand years ago (ka)), which coincides with a northern range expansion after the Last Glacial Maximum (about $22 \mathrm{ka}$ ).
Atlantic genotypes with higher levels of Midwest introgressions exhibited a more-upland suite of traits (Fig. 4b) and were overrepresented along the northern margin of the otherwise subtropical and temperate range of the Atlantic subpopulation (Fig. 4c). Consistent with adaptive roles for genomic introgressions in other systems ${ }^{31,37}$, these findings suggest that introgression of putatively northern-adapted alleles from the Midwest into the Atlantic subpopulation could have facilitated the post-glacial colonization by switchgrass of colder habitats in the northeastern coastal region of the USA. To test this hypothesis, we conducted redundancy analyses to relate the presence of introgression blocks with climatic, geographical and phenotypic factors. Overall, Midwest introgressions in the Atlantic subpopulation were over four times more strongly associated with climate (percentage of variance explained $=46.5 \%$ ) than geography (11.5\%). Although 532 and 651 introgressions from the Midwest to the Atlantic subpopulation were associated with climate of origin or biomass, respectively, 254 introgressions were outliers for both analyses-representing a nearly 7-fold enrichment over expectations of independence between each set (odds ratio $=6.99, P<1 \times 10^{-15}$ ). These results reinforce the hypothesis that Midwest introgressions have shaped the climatic niche and phenotypic distribution of the northern Atlantic genotypes and support a growing body of evidence that demonstrates that adaptive introgressions can facilitate both range expansion and ecotype evolution ${ }^{38,39}$.

\section{Reduced heritability of dominant subgenomes}

Polyploidy is common among lineages of flowering plants and can increase the genetic diversity available to selection ${ }^{40,41}$, which can lead to adaptive evolution or sorting that alters ecological niche characteristics ${ }^{42}$. This process may explain the generally greater prevalence of polyploids in poleward latitudes and higher elevations that were once covered by ice sheets during glacial cycles ${ }^{43}$.

Genes duplicated during the formation of a polyploid can subfunctionalize (divide ancestral gene functions among paralogous genes), neofunctionalize (evolve new gene function for paralogues) or simply be lost ${ }^{44}$. Following polyploid speciation, one subgenome commonly retains more genes and exhibits, on average, higher expression levels than the other subgenome, a phenomenon known as subgenome dominance ${ }^{45}$. As with other polyploids ${ }^{46-48}$, subgenome dominance and subfunctionalization were clear in switchgrass. Relative to the 
N subgenome, the K subgenome had higher gene density (77.4 versus 68.0 genes per $\mathrm{Mb}$, binomial $\left.P<1 \times 10^{-15}\right)$, more upregulated genes $(5,445$ versus 4,477 , binomial $P<1 \times 10^{-15}$ ) and lower rates of mutation accumulation $(5,255$ genes in the $K$ subgenome with a synonymous mutation rate $\left(K_{\mathrm{s}}\right)$ greater than that in the N subgenome, versus 6,751 genes in the $\mathrm{N}$ subgenome with $K_{\mathrm{s}}$ greater than that in the $\mathrm{K}$ subgenome, binomial $P<1 \times 10^{-15}$ ). Combined, all 11 of our subgenome statistics (Extended Data Fig. 4) point to stronger evolutionary constraint of and bias towards the K subgenome, which suggests that the potential for adaptive evolution may be differentially partitioned between subgenomes.

Given the evolutionary biases towards retention of the K subgenome, we expected to see stronger signals of climate adaptation ${ }^{44}$, biomass and survival among SNPs on the K subgenome. Instead, $75.9 \%$ of biomass SNP-heritability was attributable to the N subgenome, and only 24.1\% to the $K$ subgenome across the 10 common gardens (Extended Data Fig. 4). Furthermore, 54.3\% of Midwest introgressions into the Atlantic subpopulation were found on the N subgenome, a significant enrichment (binomial test $P<1 \times 10^{-7}$ ), even when correcting for the $7.5 \%$ expansion of the $\mathrm{N}$ subgenome (binomial test $P=0.0012$ ). The abundance of introgressions and heritable biomass variation attributable to the N subgenome may appear to be at odds with subgenome evolutionary biases towards the $\mathrm{K}$ subgenome. One potential explanation for this counterintuitive finding is that relaxed evolutionary constraint (reduced purifying selection) on the $\mathrm{N}$ subgenome may have allowed for accumulation of adaptive genetic variation through directional or diversifying selection. As such, the $\mathrm{N}$ subgenome has accumulated heritable variation ${ }^{49}$ that future breeding regimes can target to shape natural switchgrass populations and improve biofuel yield.

\section{Discussion}

As the climate and the natural environment change, it is increasingly critical to qualify expectations of genetic improvements in domesticated species and the adaptive potential of wild populations ${ }^{50}$. Indeed, plant genomes offer glimpses into the past and future of crop and wild plant populations. Adaptation to glacial-interglacial cycles offers an instructive analogue for current and future environmental change, one that we explore here to investigate the past, present and future genomic mechanisms of climate adaptation and yield improvement in switchgrass.

However, the complexity of plant genomes has also presented a major barrier to the development of genetic resources that facilitate fast and effective molecular breeding. Our methodology and success in sequencing the complex genome of switchgrass will facilitate ecological and agricultural genomics in nearly any system. For example, our results demonstrate that adaptation to northern climates has been facilitated by introgressions between anciently diverged subpopulations, which provides further support for the hypothesis that admixture between divergent genomes can enhance adaptation to novel environments ${ }^{37}$. Such adaptive introgressions and heritable subgenome-specific genetic variation ${ }^{49}$ may provide the genetic paths of least resistance that permit colonization of novel habitats during periods of environmental variability. Combined, obligate outcrossing and polyploidy-traits that are often consciously avoided when selecting genomic study systems-are the primary drivers of switchgrass adaptation in nature and the sources of genetic variation available for selection to improve biofuel yield through a changing future.

\section{Online content}

Any methods, additional references, Nature Research reporting summaries, source data, extended data, supplementary information, acknowledgements, peer review information; details of author contributions and competing interests; and statements of data and code availability are available at https://doi.org/10.1038/s41586-020-03127-1.
1. Lobell, D. B., Schlenker, W. \& Costa-Roberts, J. Climate trends and global crop production since 1980. Science 333, 616-620 (2011).

2. Challinor, A. J. et al. A meta-analysis of crop yield under climate change and adaptation. Nat. Clim. Chang. 4, 287-291 (2014).

3. Rosenzweig, $C$. et al. Assessing agricultural risks of climate change in the 21st century in a global gridded crop model intercomparison. Proc. Natl Acad. Sci. USA 111, 3268-3273 (2014).

4. Porter, J. R. et al. in Climate Change 2014: Impacts, Adaptation, and Vulnerability. Part A: Global and Sectoral Aspects (Contribution of Working Group II to the Fifth Assessment Report of the Intergovernmental Panel on Climate Change) (eds Field, C. B. et al.) 485-533 (Cambridge Univ. Press, 2014).

5. Bevan, M. W. et al. Genomic innovation for crop improvement. Nature 543, 346-354 (2017).

6. Nelson, R., Wiesner-Hanks, T., Wisser, R. \& Balint-Kurti, P. Navigating complexity to breed disease-resistant crops. Nat. Rev. Genet. 19, 21-33 (2018).

7. Risser, P. G., Birney, E. C. \& Blocker, H. D. The True Prairie Ecosystem (Dowden, Hutchinson and Ross, 1981)

8. Suyker, A. E. \& Verma, S. B. Year-round observations of the net ecosystem exchange of carbon dioxide in a native tallgrass prairie. Glob. Change Biol. 7, 279-289 (2001).

9. Schmer, M. R., Vogel, K. P., Mitchell, R. B. \& Perrin, R. K. Net energy of cellulosic ethanol from switchgrass. Proc. Natl Acad. Sci. USA 105, 464-469 (2008).

10. Palik, D. J., Snow, A. A., Stottlemyer, A. L., Miriti, M. N. \& Heaton, E. A. Relative performance of non-local cultivars and local, wild populations of switchgrass (Panicum virgatum) in competition experiments. PLoS ONE 11, e0154444 (2016).

11. McLaughlin, S. et al. in Perspectives on New Crops and New Uses (ed. Janick, J.) 282-299 (ASHS, 1999).

12. Vogel, K. P., Schmer, M. R. \& Mitchell, R. B. Plant adaptation regions: ecological and climatic classification of plant materials. Rangeland Ecol. Manag. 58, 315-319 (2005).

13. Casler, M. D. et al. Latitudinal and longitudinal adaptation of switchgrass populations. Crop Sci. 47, 2249-2260 (2007).

14. Lipka, A. E. et al. Accelerating the switchgrass (Panicum virgatum L.) breeding cycle using genomic selection approaches. PLoS ONE 9, e112227 (2014).

15. Poudel, H. P., Sanciangco, M. D., Kaeppler, S. M., Buell, C. R. \& Casler, M. D. Genomic prediction for winter survival of lowland switchgrass in the northern USA. G3 9, 1921-1931 (2019).

16. Lowry, D. B. et al. QTL x environment interactions underlie adaptive divergence in switchgrass across a large latitudinal gradient. Proc. Natl Acad. Sci. USA 116, 12933-12941 (2019).

17. Triplett, J. K., Wang, Y., Zhong, J. \& Kellogg, E. A. Five nuclear loci resolve the polyploid history of switchgrass (Panicum virgatum L.) and relatives. PLoS ONE 7, e38702 (2012).

18. Martínez-Reyna, J. M. \& Vogel, K. P. Incompatibility systems in switchgrass. Crop Sci. 42. 1800-1805 (2002).

19. Casler, M. D., Vogel, K. P. \& Harrison, M. Switchgrass germplasm resources. Crop Sci. 55 2463-2478 (2015).

20. Evans, J. et al. Extensive genetic diversity is present within North American switchgrass germplasm. Plant Genome 11, 1-16 (2018).

21. Wang, T., Hamann, A., Spittlehouse, D. \& Carroll, C. Locally downscaled and spatially customizable climate data for historical and future periods for North America. PLoS ONE 11, e0156720 (2016).

22. Fick, S. E. \& Hijmans, R. J. WorldClim 2: new 1-km spatial resolution climate surfaces for global land areas. Int. J. Climatol. 37, 4302-4315 (2017).

23. Lowry, D. B. et al. Adaptations between ecotypes and along environmental gradients in Panicum virgatum. Am. Nat. 183, 682-692 (2014).

24. Casler, M. D., Vogel, K. P., Taliaferro, C. M. \& Wynia, R. L. Latitudinal adaptation of switchgrass populations. Crop Sci. 44, 293-303 (2004)

25. Porter, C. L. An analysis of variation between upland and lowland switchgrass Panicum virgatum L in central Oklahoma. Ecology 47, 980-992 (1966).

26. McMillan, C. Ecotypic differentiation within four North American prairie grasses. I. Morphological variation within transplanted community fractions. Am. J. Bot. $\mathbf{5 1}$ 1119-1128 (1964).

27. Grabowski, P. P., Morris, G. P., Casler, M. D. \& Borevitz, J. O. Population genomic variation reveals roles of history, adaptation and ploidy in switchgrass. Mol. Ecol. 23, 4059-4073 (2014).

28. Lu, F. et al. Switchgrass genomic diversity, ploidy, and evolution: novel insights from a network-based SNP discovery protocol. PLoS Genet. 9, e1003215 (2013).

29. Casler, M. D. et al. 30 years of progress toward increased biomass yield of switchgrass and big bluestem. Crop Sci. 58, 1242-1254 (2018).

30. Casler, M. D. \& Vogel, K. P. Selection for biomass yield in upland, lowland, and hybrid switchgrass. Crop Sci. 54, 626-636 (2014).

31. Suarez-Gonzalez, A., Lexer, C. \& Cronk, Q. C. B. Adaptive introgression: a plant perspective. Biol. Lett. 14, 20170688 (2018).

32. Urbut, S. M., Wang, G., Carbonetto, P. \& Stephens, M. Flexible statistical methods for estimating and testing effects in genomic studies with multiple conditions. Nat. Genet. 51, 187-195 (2019).

33. Kass, R. E. \& Raftery, A. E. Bayes factors. J. Am. Stat. Assoc. 90, 773-795 (1995).

34. Fournier-Level, A. et al. A map of local adaptation in Arabidopsis thaliana. Science 334, 86-89 (2011).

35. Zhang, Y. et al. Post-glacial evolution of Panicum virgatum: centers of diversity and gene pools revealed by SSR markers and cpDNA sequences. Genetica 139, 933-948 (2011).

36. Corbett-Detig, R. \& Nielsen, R. A hidden Markov model approach for simultaneously estimating local ancestry and admixture time using next generation sequence data in samples of arbitrary ploidy. PLoS Genet. 13, e1006529 (2017).

37. Todesco, M. et al. Massive haplotypes underlie ecotypic differentiation in sunflowers. Nature 584, 602-607 (2020).

38. Lucek, K., Lemoine, M. \& Seehausen, O. Contemporary ecotypic divergence during a recent range expansion was facilitated by adaptive introgression. J. Evol. Biol. 27, 2233-2248 (2014).

39. Whitney, K. D. et al. Quantitative trait locus mapping identifies candidate alleles involved in adaptive introgression and range expansion in a wild sunflower. Mol. Ecol. 24 2194-2211 (2015). 


\section{Article}

40. Comai, L. The advantages and disadvantages of being polyploid. Nat. Rev. Genet. 6 836-846 (2005)

41. Mattenberger, F., Sabater-Muñoz, B., Toft, C. \& Fares, M. A. The phenotypic plasticity of duplicated genes in Saccharomyces cerevisiae and the origin of adaptations. G3 7, 63-75 (2017).

42. Clark, J. W. \& Donoghue, P. C. J. Whole-genome duplication and plant macroevolution. Trends Plant Sci. 23, 933-945 (2018).

43. Stebbins, G. L. Polyploidy, hybridization, and the invasion of new habitats. Ann. Mo. Bot. Gard. 72, 824 (1985).

44. Bird, K. A., VanBuren, R., Puzey, J. R. \& Edger, P. P. The causes and consequences of subgenome dominance in hybrids and recent polyploids. New Phytol. 220, 87-93 (2018).

45. Flagel, L. E. \& Wendel, J. F. Evolutionary rate variation, genomic dominance and duplicate gene expression evolution during allotetraploid cotton speciation. New Phytol. 186, 184-193 (2010).

46. Edger, P. P. et al. Origin and evolution of the octoploid strawberry genome. Nat. Genet. 51, 541-547 (2019).

47. Chen, Z. J. et al. Genomic diversifications of five Gossypium allopolyploid species and their impact on cotton improvement. Nat. Genet. 52, 525-533 (2020).

48. Session, A. M. et al. Genome evolution in the allotetraploid frog Xenopus laevis. Nature 538, 336-343 (2016)

49. Nieto Feliner, G., Casacuberta, J. \& Wendel, J. F. Genomics of evolutionary novelty in hybrids and polyploids. Front. Genet. 11, 792 (2020).

50. Davis, M. B. \& Shaw, R. G. Range shifts and adaptive responses to Quaternary climate change. Science 292, 673-679 (2001).

51. South, A. rnaturalearthdata: World Vector Map Data from Natural Earth Used in 'rnaturalearth'. R package version 0.1.0. https://CRAN.R-project.org/ package=rnaturalearthdata $(2017)$.

Publisher's note Springer Nature remains neutral with regard to jurisdictional claims in published maps and institutional affiliations.

pen Access This article is licensed under a Creative Commons Attribution 4.0 International License, which permits use, sharing, adaptation, distribution and reproduction in any medium or format, as long as you give appropriate credit to the original author(s) and the source, provide a link to the Creative Commons license and indicate if changes were made. The images or other third party material in this article are included in the article's Creative Commons license, unless indicated otherwise in a credit line to the material. If material is not included in the article's Creative Commons license and your intended use is not permitted by statutory regulation or exceeds the permitted use, you will need to obtain permission directly from the copyright holder. To view a copy of this license, visit http://creativecommons.org/licenses/by/4.0/.
${ }^{1}$ Genome Sequencing Center, HudsonAlpha Institute for Biotechnology, Huntsville, AL, USA. ${ }^{2}$ Department of Integrative Biology, University of Texas at Austin, Austin, TX, USA ${ }^{3}$ Department of Energy Joint Genome Institute, Lawrence Berkeley National Laboratory, Berkeley, CA, USA. ${ }^{4}$ Department of Molecular and Cell Biology, University of California, Berkeley, Berkeley, CA, USA. ${ }^{5}$ Department of Plant Biology, Rutgers University, New Brunswick, NJ, USA. ${ }^{6}$ Plant Genetic Resources Conservation Unit, USDA-ARS, Griffin, GA, USA. ${ }^{7}$ Department of Plant Biology, Michigan State University, East Lansing, MI, USA. ${ }^{8}$ Arizona Genomics Institute, University of Arizona, Tucson, AZ, USA. ${ }^{9}$ Institute of Plant Breeding, Genetics and Genomics, University of Georgia, Athens, GA, USA. ${ }^{10}$ Department of Crop and Soil Sciences, University of Georgia, Athens, GA, USA. "Department of Plant Biology, University of Georgia, Athens, GA, USA. ${ }^{12}$ Department of Plant and Environmental Sciences, Clemson University, Clemson, SC, USA. ${ }^{13}$ Department of Biological Sciences, Marshall University, Huntington, WV, USA. ${ }^{14}$ School of Biotechnology, Jawaharlal Nehru University, New Delhi, India. ${ }^{15}$ School of Computational and Integrative Sciences, Jawaharlal Nehru University, New Delhi, India. ${ }^{16}$ Noble Research Institute LLC, Ardmore, OK, USA. ${ }^{17}$ Department of Agronomy and Horticulture, University of Nebraska, Lincoln, NE, USA. ${ }^{18}$ Department of Agronomy, Horticulture and Plant Science, South Dakota State University, Brookings, SD, USA. ${ }^{19}$ Grassland, Soil and Water Research Laboratory, USDA-ARS, Temple, TX, USA. ${ }^{20}$ Division of Plant Sciences, University of Missouri, Columbia, MO, USA. ${ }^{21}$ Environmental Science Division, Argonne National Laboratory, Lemont, IL, USA. ${ }^{22}$ Kika de la Garza Plant Materials Center, USDA-NRCS, Kingsville, TX, USA. ${ }^{23}$ Plant Breeding Department, Antonio Narro Agrarian Autonomous University, Saltillo, Mexico. ${ }^{24}$ Wheat, Sorghum, and Forage Research Unit, USDA-ARS, Lincoln, NE, USA. ${ }^{25}$ Texas A\&M AgriLife Research and Extension Center, Texas A\&M University, Overton, TX, USA. ${ }^{26}$ Department of Plant Pathology and the Genome Center, University of California, Davis, Davis, CA, USA ${ }^{27}$ Joint BioEnergy Institute, Emeryville, CA, USA. ${ }^{28}$ Western Regional Research Center, USDA-ARS, Albany, CA, USA. ${ }^{29}$ Department of Plant and Soil Sciences, Oklahoma State University, Stillwater, OK, USA. ${ }^{30}$ Department of Microbiology and Plant Biology, University of Oklahoma, Norman, OK, USA. ${ }^{31}$ Institute of Biological Chemistry, Washington State University, Pullman, WA, USA. ${ }^{32}$ US Dairy Forage Research Center, USDA-ARS, Madison, WI, USA. ${ }^{33}$ DOE Great Lakes Bioenergy Research Center, University of Wisconsin, Madison, WI, USA. ${ }^{34}$ DOE Center for Bioenergy Innovation, Oak Ridge, TN, USA. ${ }^{35}$ DOE Great Lake Bioenergy Research Center, Michigan State University, East Lansing, MI, USA. ${ }^{36}$ Center for Advanced Bioenergy and Bioproducts Innovation, Berkeley, CA, USA. ${ }^{37}$ Chan-Zuckerberg Biohub, San Francisco, CA, USA. ${ }^{38}$ These authors contributed equally: John T. Lovell, Alice H. MacQueen, Sujan Mamidi, Jason Bonnette, Jerry Jenkins. ${ }^{\circledR}$ e-mail: jlovell@hudsonalpha. org; tjuenger@mail.utexas.edu; jschmutz@hudsonalpha.org

(c) The Author(s) 2021 


\section{Methods}

No statistical methods were used to predetermine sample size. The experiments were completely randomized, and investigators were not aware of genotype identifiers while conducting experiments or sequencing.

\section{Plant collections, propagation, cultivation and phenotyping}

To form the diversity panel, seeds, rhizomes and clonal propagules from natural and common garden sources were collected from 2010 to 2018. Plants grown from seed followed a standard growth procedure $^{16}$. In brief, $10-15$ seeds were sown in $9-\mathrm{cm}$ square pots containing a mixture of ProMix BX potting soil (Premier Tech Horticulture) and Turface MVP calcined clay (Turface Athletics) and vernalized for 7 days at $4{ }^{\circ} \mathrm{C}$. Pots were then placed in a lit greenhouse with 14 -h day length and $30-{ }^{\circ} \mathrm{C} / 22-{ }^{\circ} \mathrm{C}$ day/night temperature. Seedlings were thinned at the 3-leaf stage to 1 plant per pot and allowed to grow until the 5-tiller stage. Rhizome propagules and 5-tiller seedlings were transferred to 5-gallon pots containing finely ground pine bark mulch (Lone Star Mulch) and time-release fertilizer (Osmocote14-14-14, ScottsMiracleGro). All individual plants were propagated in Austin by clonal division from 2016 to 2018 , targeting $>10$ clones per unique accession. Cleary $3336 \mathrm{~F}$ systemic fungicide (Cleary Chemicals) was applied to the plants as necessary to control fungal pathogens. Plants were placed in 1-gallon pots for the final propagation.

Planting in the field sites occurred from 15 May to 10 July 2018 and followed previously published methods ${ }^{16}$. In brief, plants were transported to each site by truck, where each field was covered with one layer of DeWitt weed cloth. Plants were placed in holes that were cut into the weed cloth into a honeycomb design in which each plant had four nearest neighbours, all located $1.56 \mathrm{~m}$ from one another. To prevent edge effects, the lowland Blackwell cultivar was planted at every edge position. Plants were hand-watered following transplantation. Aboveground portions of all plants were left to stand over the winter of 2018-2019 and removed in the spring of 2019 before spring tiller emergence. At the end of the 2019 season, plants were tied upright as a bunch and harvested with sickle bar mowers.

We generated two measures of fitness for the 2019 growing season: log-transformed biomass $(\mathrm{kg})$ and proportion of winter survival (Supplementary Data 8). Biomass data were obtained from all living individuals during harvest in October and November 2019. Plants with an estimated mass $<750 \mathrm{~g}$ were placed in paper bags and dried whole at $60^{\circ} \mathrm{C}$ until no additional moisture loss occurred, then weighed for total dry biomass. Plants with an estimated mass $>750 \mathrm{~g}$ were weighed in the field for wet biomass on a hanging scale with $\mathrm{a} \pm 5$-g resolution. To determine biomass of these plants, approximately $500 \mathrm{~g}$ of whole tillers were subsampled from each plant, weighed, dried as above and reweighed. The wet biomass of the whole-plant sample was then multiplied by the per cent moisture in the subsample to approximate total dry biomass. Plants were considered to have experienced winter mortality during the 2018-2019 winter season when no new growth was seen from plant crowns by 1 June 2019 . The dead plant crowns were excised from the experiment and replaced with plants of the Blackwell cultivar in July or September 2019.

\section{Genome assembly and polishing}

We sequenced the Alamo switchgrass genotype AP13 using a whole genome shotgun sequencing strategy and standard sequencing protocols at the Department of Energy Joint Genome Institute and the HudsonAlpha Institute for Biotechnology. The genome was assembled and polished from 4,520,785 PacBio reads (121.66 $\times$ raw sequence coverage from a total of 59 P6C4 2.0 and 2.1 chemistry cells with 10 -h movie times and a p-read yield of $91.76 \mathrm{~Gb}$ ) (Extended Data Fig. 1) using the MECAT assembler ${ }^{52}$ and ARROW polisher ${ }^{53}$. Final genome polishing and error correction was conducted with one 400 bp insert $2 \times 150$ bp Illumina
HiSeq fragment library $(177.1 \times)$. Reads with $>95 \%$ simple sequence repeats and reads $<50$ bp after trimming for adaptor and quality $(q<20$, 5 -bp window average) were removed. The final read set consisted of $1,259,053,614$ reads for a total of $168 \times$ coverage of high-quality Illumina bases. This produced an initial diploid assembly of 6,600 scaffolds (6,600 contigs), with a contig $\mathrm{N}_{50}$ of $1.1 \mathrm{Mb}, 3,489$ scaffolds larger than $100 \mathrm{~kb}$ and a total $2 \mathrm{C}$ (diploid) genome size of 2,013.4 Mb.

Assembling a haploid genome in an outbred individual, such as AP13, will generally yield both haploid copies in heterozygous regions, necessitating computational steps to represent each chromosome as a single-copy haplotype without duplicate copies being unnecessarily repeated. Our initial assembly was approximately double the expected haploid (1C) genome size of $1.2 \mathrm{~Gb}$. Therefore, to detect putative meiotically homologous haplotypes, we identified and counted shared 24-mers that occurred exactly twice in the assembly and binned contigs accordingly. A total of 3,152 shorter and redundant alternative haplotypes and 2,387 overlapping contig ends were identified, comprising a total sequence of $871.2 \mathrm{Mb}$. The remaining $1,142.2 \mathrm{Mb}$ of sequence was ordered and oriented into 18 chromosomes by aligning genetic markers from 2 available maps (Supplementary Data 1 ) to the MECAT assembly; 563 joins and 57 breaks were made, with 10,000 Ns representing the unsized gap sequence. Overall, $97.2 \%$ of the assembled sequence was contained in the chromosomes. Telomeric sequence was identified using the (TTTAGGG) ${ }_{n}$ repeat and properly oriented. The remaining scaffolds were screened against GenBank bacterial proteins and organelle sequences and removed if found to match these sequences. To resolve minor overlapping regions on contig ends, adjacent contig ends were aligned to one another using $\mathrm{BLAT}^{54}$; a total of 47 adjacent duplicate contig pairs were collapsed.

We conducted two rounds of error correction. First, we corrected homozygous SNPs and insertions and/or deletions (indels) by aligning the Illumina $2 \times 150$ bp library to the release consensus sequence using bwa mem ${ }^{55}$ and identifying homozygous SNPs and indels with the UnifiedGenotyper tool of GATK ${ }^{56}$. A total of 690 homozygous SNPs and 80,199 homozygous indels were corrected in the release. Second, we computationally finished 11,343 assembled contigs sequenced from $\mathrm{BAC}$ clones with a combination of $\mathrm{ABI} 3730 \mathrm{XL}$ capillary sequenc$\mathrm{ers}^{57}$ and single index Illumina clone pools and aligned this set of switchgrass clones to the SNP-fixed genome to find heterozygous SNPs that were out of phase with their neighbours. To resolve these phase-switched alleles, the full set of the raw PacBio reads was aligned to the assembly. For each read, the phase of each heterozygous site was determined and 62,732 out-of-phase heterozygous sites were corrected.

To distinguish the $\mathrm{N}$ and $\mathrm{K}$ subgenomes, we used a de novo repeat-clustering method and validated this with phylogenetic distances to a related species. We searched for 'diagnostic' 15-mers via Jellyfish ${ }^{58}$ in LTR regions of Gypsy, Copia and Pao insertions (identified by RepeatMasker ${ }^{59}$ and LTRHarvest ${ }^{60}$ ) that distinguished each set of homologous chromosomes ( $\leq 1$ hit in one homologue and $\geq 100$ in the other). The LTR sequences that shared common 15-mers were grouped as superfamilies and were aligned within each superfamily by BLAST. Superfamily members with significant BLAST hits ( $e<0.01, \geq 90 \%$ length) were assigned into families and aligned by Mafft ${ }^{61}$. Jukes-Cantor distances between LTR families were computed by the R ape package ${ }^{62}$, and clustered into two distinct sets of subgenomes. Clustering was identical between LTRs and alignments to P. rudgei (K.M.D. and E. Kellogg, unpublished data), which is an ancient relative of the K subgenome ${ }^{17}$, giving high confidence that we have effectively assigned all chromosomes to the correct subgenomes. Finally, we assigned chromosome identifiers and oriented each chromosome pseudomolecule via synteny with Setaria italica ${ }^{63}$. The final haploid version 5.0 release contained 1,125.2 Mb of sequence, consisting of 626 contigs with a contig $\mathrm{N} 50$ of $5.5 \mathrm{Mb}$ and a total of $97.2 \%$ of assembled bases in chromosomes. 


\section{Gene annotation}

Transcript assemblies were made from about 2 billion pairs of $2 \times 150$-bp stranded paired-end Illumina RNA-seq reads, about 1 billion pairs of $2 \times 100$-bp paired-end Illumina RNA-seq reads and 454 reads (Supplementary Data 3 ) using PERTRAN (details of which have previously been published $\left.{ }^{64}\right)$. In brief, PERTRAN conducts genome-guided transcriptome short-read assembly via $\mathrm{GSNAP}^{65}$ and builds splice alignment graphs after alignment validation, realignment and correction. In total, around 4.5 million PacBio Iso-Seq circular consensus sequences ${ }^{66}$ were corrected and collapsed, resulting in approximately 677,000 putative full-length transcript assemblies. Subsequently, 668,176 transcript assemblies were constructed using PASA $^{67}$ from RNA-seq reads, full-length cDNA, Sanger expressed sequence tags, and corrected and collapsed PacBio circular consensus sequence reads. Loci were determined by EXONERATE ${ }^{68}$ alignments of switchgrass transcript assemblies and proteins from Arabidopsis thaliana ${ }^{69}$, soybean $^{70}$, Kitaake rice $^{71}$, Setaria viridis $^{72}$, P. hallii var. hallii ${ }^{64}$, Sorghum bicolor $^{73}$, Brachypodium $^{2}$ distachyon ${ }^{74}$, grape and Swiss-Prot ${ }^{75}$ proteomes. These alignments were accomplished against a repeat-soft-masked switchgrass genome using RepeatMasker $^{59}$ (repeat library from RepeatModeler ${ }^{76}$ and RepBase ${ }^{77}$ ) with up to 2,000-bp extension on both ends unless extending into another locus on the same strand. Incomplete gene models, which had low homology support without full transcriptome support, or short single exon genes (<300-bp coding DNA sequences (CDS)) without protein domain or good expression were removed.

\section{Comparative genomics}

Syntenic orthologues and paralogues were inferred for the two switchgrass subgenomes via the GENESPACE pipeline ${ }^{64}$, using default parameters and two outgroups: P. hallii var. hallii ${ }^{64}$ and S. bicolor $^{73}$. In brief, GENESPACE parses protein similarity scores into syntenic blocks and runs orthofinder ${ }^{78}$ on synteny-constrained blast results. The resulting block coordinates and syntenic orthology networks give high-confidence anchors for evolutionary inference.

To calculate the ancestral states of CDS regions, we first determined sequences that share common ancestry using genomes from Phytozome $^{79}$. The final number of hits to the switchgrass genome were 38,960 and 33,772 for $P$. hallii, and S. bicolor, respectively. For any given orthology network, we built two multiple sequence alignments in mafft ${ }^{61}$, one excluding the focal switchgrass sequence $\left(\mathrm{msa}_{0}\right)$ and one forcing $\mathrm{msa}_{0}$ to align to the coordinate system of the focal sequence via the --keeplength parameter. We then extracted marginal character states with the maximum likelihood algorithm in Phangorn ${ }^{80}$. For each reconstruction, only the internal node closest to the switchgrass branch was used as the ancestral state. Overall, we analysed 40,943 switchgrass gene models (216,157 exons) covering 54.95 Mb (Supplementary Data 9).

\section{Subgenome evolution and dating}

To infer the ages of the subgenomes and tetraploid switchgrass, we took a conservative set of orthologues with simple 2:1:1 networks between P.virgatum, P. hallii and S. italica. This yielded 45,045 switchgrass proteins aligning to 24,549 $P$. hallii proteins, resulting in 20,496 homologue pairs and 4,053 singletons (2,396 for K subgenome and 1,660 for N subgenome) from the cross-species analysis. We aligned the translated CDS of these sequences using Dialign-TX ${ }^{81}$. The aligned CDS sequences were concatenated and fed to Gblocks ${ }^{82}$ using default parameters. Gblocks filtered the alignment of $18,044,244$ CDS nucleotides to $16,321,302$ positions, in 50,334 blocks. The resulting alignment was then used in $\mathrm{PhyML}^{83}$ to build a maximum-likelihood tree using the general-time reversible model. This tree was used as an input to $r 8 \mathrm{~s}^{84}$, to compute a time tree and calibrate the Panicum-Setaria node of the tree to $13.1 \mathrm{Ma}^{63}$. To date subgenome divergence and therefore the timing of polyploid switchgrass speciation, we leveraged burst distances, which refer to all distances within an LTR family (whereas pairwise distances refer to the distance between the $5^{\prime}$ and $3^{\prime}$ LTRs of the same insertion). The $5^{\prime}$ versus $3^{\prime}$ distances of the $\mathrm{N}$ - or K-subgenome-specific retrotransposons were used to date the insertion times of those elements. This method cannot be used for the P.virgatum-specific or Panicum-specific families because the more recent expansions of those elements dominate the distributions. Instead, we relied on comparing the best cross-species alignments to estimate the LTR distances of the $P$. virgatum-P. hallii and Panicum-Setaria nodes. This way, we have calibration points to compare the LTR distances to the more confident protein-coding gene divergences between species.

\section{Subfunctionalization and gene expression analyses}

To assess whether the subgenome evolution biases observed at the protein-coding sequence scale were manifest in phenotypes, we explored gene expression biases between homologues from biologically replicated AP13 leaf tissue $(n \geq 5)$ collected at two sites ( $\mathrm{TX}_{2}$ and $\mathrm{MI})$. Illumina paired-end RNA-seq 150 -bp reads were quality trimmed $(Q \geq 25)$ and reads shorter than $50 \mathrm{bp}$ after trimming were discarded. High-quality sequences were aligned to $P$. virgatum $v 5.1$ reference genome using GSNAP ${ }^{65}$ and counts of reads uniquely mapping to annotated genes were obtained using HTSeq v.0.11.2 ${ }^{85}$. The test for differential expression was conducted through a likelihood ratio test in DESeq $2^{86}$. Library sizes were calculated before splitting the reads by subgenome; these sizes were used as the size factors in the analysis of differential expression. Subfunctionalization was defined as a significant subgenome-by-environment interaction from the likelihood ratio test. Subgenome expression bias was tested for both the field gardens and annotation libraries using post hoc Wald-test contrasts between subgenomes within conditions. Significant bias was defined as differential expression false-discovery-rate-adjusted $P<0.05$. Weighted gene coexpression clustering of AP13 gene annotation RNA-seq libraries was conducted with WGCNA ${ }^{87}$ with a power of 6 . Raw counts can be found in Supplementary Data 10.

\section{Ploidy assessment}

We used a LSRFortessa SORP Flow Cytometer (BD Biosciences) to determine ploidy levels of the resequenced accessions. For each plant, 200-300 mg of young leaf tissue was macerated in a Petri dish with a razor blade and treated for $15 \mathrm{~min}$ with $1 \mathrm{ml}$ Cystain PI Absolute P nuclei extraction buffer (Sysmex Flow Cytometry) mixed with $1 \mu \mathrm{l}$ 2-mercaptoethanol. Samples were filtered to isolate free nuclei with a CellTrics 30- $\mu \mathrm{m}$ filter (Sysmex) and treated for $20 \mathrm{~min}$ on wet ice with $2 \mathrm{ml}$ of Cystain PI Absolute P staining buffer (Sysmex), $12 \mu \mathrm{l}$ of propidium iodide and $6 \mu \mathrm{l}$ of RNase A. Samples were run on the flow cytometer to determine nuclei size with a minimum of 10,000 nuclei analysed per sample. Output from the flow cytometer was analysed with FlowJo software (BD Biosciences) and samples were binned into three categories on the basis of the average units of fluorescence per nuclei (Supplementary Fig. 1). Ploidy level of the sample was considered $4 \times$ if the cell population had 40,000-80,000 units of fluorescence, $6 \times$ for $80,000-100,000$ units and $8 \times$ for $100,000-140,000$ units. The binning parameters were established with flow cytometry data from several $P$. virgatum accessions of known ploidy.

We also assessed ploidy of the samples via the distribution of variant allele frequency at biallelic SNPs (as described in 'Variant calling'). This method assumes that tetraploids and octoploids follow different allele frequency distribution patterns, with tetraploids having $0.5 / 0.5$ (reference and variant depths) and octoploids having a mixture of $0.75 / 0.25$ and $0.5 / 0.5$. If the proportion of hits with $0.48 \leq x \leq 0.52$ was $<0.035$, the library was considered octoploid and if it was $\geq 0.035$, tetraploid; 837 out of 870 samples $(96.2 \%)$ that had flow cytometry data matched with these results.

\section{Variant calling}

A total of 789 tetraploid diversity samples were resequenced at a median depth of $59 \times($ range $20 \times-140 \times$ ). Of these, 732 were used for further 
analysis after filtering for missing data, outlier elevated heterozygosity and collection site discrepancies. The samples were sequenced using Illumina HiSeq X10 and Illumina NovaSeq 6000 paired-end sequencing $(2 \times 150 \mathrm{bp})$ at HudsonAlpha Institute for Biotechnology and the Joint Genome Institute. To account for different library sizes, reads were pruned to $\leq 50 \times$ coverage, then mapped to the $v 5$ assembly using bwa-mem ${ }^{55}$.

SNPs were called by aligning Illumina reads to the AP13 reference with BWA-mem. The resulting.bam file was filtered for duplicates using Picard (http://broadinstitute.github.io/picard) and realigned around indels using GATK $3.0^{56}$. Multi-sample SNP calling was done using SAMtools mpileup ${ }^{88}$ and Varscan $V 2.4 .0^{89}$ with a minimum coverage of eight and a minimum alternate allele count of four. Genotypes were called via a binomial test. SNPs within 25 bp of a $24-$ mer repeat were removed from further analyses. Only SNPs with $\leq 20 \%$ missing data and minor allele frequencies $>0.005$ were retained, resulting in $33,905,042$ SNPs across $75 \%$ of the genome at a coverage depth between $8 \times$ and $500 \times$. Phasing was performed using SHAPEIT $3^{90} . F_{\mathrm{ST}}$ calculations were accomplished via vcftools ${ }^{91}$. We tested for subgenome read-mapping bias by generating mean coverage per $\mathrm{Mb}$ for each of the 732 libraries and 18 chromosomes. We then fit a mixed effects linear model to these data in Ime ${ }^{92}$ in which the chromosome number (1-9) was a random effect, to test the main effect of subgenome. Models with and without the main effect term were compared via a likelihood ratio test.

Individual de novo assemblies for the 732 short read libraries were constructed using HipMer ${ }^{93}$ with a $k$-mer size of 101 to maximize haplotype splitting among contigs. As the assemblies varied in quality and contiguity, the sample set considered for gene presence-absence and structural variant detection was narrowed to 251 samples (pan-genome set) based on total assembly size, contig $\mathrm{N}_{50}$ length and total gene alignments per library.

To assess presence-absence variation of genes across the pan-genome, we aligned all AP13 proteins and a unique set of 6,161 proteins from Oropetium thomaeum $\left(n_{\text {proteins }}=1,476\right)^{94}$, S. italica $(n=1,085)^{63}$, Setaria viridis $(n=891)^{72}$, P. hallii var. filipes $(n=1,048)^{64}$, S. bicolor $(n=878)^{95}$ and $P$. hallii var. hallii $(n=772)^{64}$. These unique genes were extracted from single-copy orthology networks inferred via orthofinder ${ }^{78}$ and selection owing to a lack of orthology to switchgrass. All proteins ( $\geq 100$ amino acids) were aligned to all de novo assemblies using BLAT $^{54}$. Gene alignments from AP13 proteins were considered present if they aligned with greater than or equal to $80 \%$ identity and $75 \%$ coverage, whereas other grass proteins were considered present with alignments greater than $70 \%$ identity and $75 \%$ coverage (to allow greater divergence among species). Variable (pan-genome shell) genes (considered present across $40-60 \%$ of the population; $n=5,432$ ) were extracted from the presence-absence variation matrix and used to visualize differences among non-admixed individuals from the Atlantic, Gulf and Midwest subpopulations. Testing genes that were significantly over-or under-represented within each subpopulation was conducted with a $\chi^{2}$ test with a Benjamini-Hochberg multiple testing correction $(P \leq 0.05)$.

To detect structural variants across the pan-genome, contigs ( $\geq 2 \mathrm{~kb})$ from each library were aligned to the AP13 reference genome using ngmlr ${ }^{96}$ with default settings for PacBio reads. The resulting .bam file was sorted using samtools ${ }^{88}$ and used for calling structural variants with sniffles ${ }^{96}$. Individual structural variant calls were merged across samples using SURVIVOR ${ }^{97}$, with a maximum allowed distance of $1 \mathrm{~kb}$. The resulting.vcf file was filtered using bcftools ${ }^{88}$ using a minimum minor allele frequency of 0.1 , and considering only insertions and deletions between 100 and $1,500 \mathrm{bp}$ in length.

\section{Population genomics}

To assess the genetic population structure of the 732 tetraploid libraries (Supplementary Data 4), we extracted all fourfold degenerate sites (putatively neutral) with ancestral state calls (Supplementary
Data 9) from the ancestral state alignments. This list of sites, which represents our highest confidence neutral loci, was then linkage-disequilibrium-pruned using a threshold of $|r| \leq 0.6$, resulting in 59,789 sites for downstream analyses in the R package SNPRelate ${ }^{98}$.

The extent of linkage disequilibrium for the population was determined from SNPs ${ }^{99}$ in PLINK ${ }^{100}$. Linkage disequilibrium $\left(r^{2}\right)$ was calculated using plink (--ld-window 500--ld-window-kb 2000). The $r^{2}$ value was averaged every $500 \mathrm{bp}$. A nonlinear model was fit for this data in Rusing the nls function, and the extent was determined as to when the linkage disequilibrium $\left(r^{2}\right)$ nonlinear curve stabilized.

Population genetic structure was assessed hierarchically. Given the presence of highly divergent ecotypes across the study range, we first analysed the broadest genetic population structure using discriminant analysis of principal components (DAPC) ${ }^{101}$ in adegenet v.2.0.1 $1^{102}$. This method does not rely on common assumptions (for example, HardyWeinberg equilibrium and linkage disequilibrium) that underlie many population clustering approaches and therefore provides a valuable tool to look at broad structural divisions. DAPC demonstrated a strong set of gene pools and separated Midwest genotypes from all others. We then evaluated the genetic population structure and potential admixture of the remaining non-Midwest individuals using a Bayesian clustering algorithm implemented in STRUCTURE v.2.3.4 ${ }^{103}$ via the admixture model with correlated allele frequencies. The analysis consisted of 20,000 burn-in steps and 30,000 replicates of 1-6 genotypic groups, each of which was run 10 times. Ancestry coefficients across all subpopulations were assigned post hoc through eigenvector decomposition in SNPRelate.

We inferred the demographic history of the switchgrass samples using Multiple Sequentially Markovian Coalescent (MSMCv.2.0 ${ }^{104}$ ), which is a population genetic method used to infer demographic history and population structure through time from sequence data. This method models an approximate version of the coalescent under recombination, and produces tests of both population size and divergence time. MSMC was run using four haplotypes for each subpopulation, skipping ambiguous sites, an estimated rhoOverMu of 0.25 and a time segment pattern of $10 \times 2+20 \times 5+10 \times 2$. We estimated rhoOverMu as 0.25 as the mean value from 100 iterations without the fixed recombination parameter for 5 sets of 4 haplotypes in each subpopulation and averaged them. To estimate scaled divergence time in generations, we assumed a mutation rate of $6.5 \times 10^{-8}$. To make estimates of initial divergence time, we compared adjacent relative cross-coalescence rate (RCCR) values (past to present) (Supplementary Data 11). If there was a decline, either at a single time segment or within contiguous segments or within two interleaved time segments $(>0.01$; observed range $0.01-0.28)$, and the following neighbours were nearly zero $(\leq 0.009$; observed range:-0.1-0.009), we considered that to be a starting point for population separation. However, if there was another decline within five time segments, we considered the latter as the start of population separation. We replicated the analyses with 16 sets of different individuals for each subpopulation contrast.

Population structure was visualized across SNPs, structural variants and presence-absence variants via eigenvector decomposition of a distance matrix. First, a Euclidean distance matrix was calculated among $0 / 1 / 2$ (reference homozygote, heterozygous, alternative homozygote) library $\times$ marker matrices for each of the three variant call types. The Euclidean matrix was then scaled and centred to remove among-library coverage variance via Gower's centred similarity matrix, implemented in the $\mathrm{R}$ package $\mathrm{MDMR}^{105}$.

\section{Ecotype classification}

Mature switchgrass accessions at or near anthesis were surveyed for 16 plant traits (leaf: length, width, length/width ratio, area, lamina thickness and lamina/midrib thickness ratio; whole plant: number of tillers, tiller height, product of tiller height $\times$ number, tiller height/count ratio, panicle height, panicle height/count ratio, leaf canopy height and tiller/ 


\section{Article}

leaf height ratio; phenology: date of green-up and date of panicle emergence) to determine ecotype identity during the summer of 2019 at the University of Texas J.J. Pickle Research Campus (PKLE; or TX TAustin, $_{2}$ Texas, USA) and Michigan State University Kellogg Biological Station (KBSM; or MI (Hickory Corners, Michigan, USA)) common gardens (see Supplementary Data 5 for detailed descriptions of these variables). The phenology measurements, including green-up (when the first green vegetative structures emerge from the rhizome crown) and panicle emergence (when the first reproductive structures emerge from the tiller), were assayed daily. Detailed leaf morphology was assessed on a representative leaf of each plant by measuring length and width (in $\mathrm{mm}$ ), midrib and lamina thickness (in $\mu \mathrm{m}$ ) (Mitutoyo 547-500S caliper) and leaf area (in $\mathrm{mm}^{2}$ ) (Licor $3100 \mathrm{C}$ leaf area meter). In addition to these quantitative traits, we also generated a qualitative upland-lowland index for both the leaf and whole-plant appearance, collected at the end of the summer 2019 in Austin ( $\mathrm{TX}_{2}$ site). Each plant characteristic was assessed on a 1-5 scale from most lowland-like to most upland-like. The established cultivars Alamo and Dacotah were used for baseline measurements of lowland and upland characters, respectively. Plant characters assessed included: tiller appearance, from thickest and most lowland-like to thinnest and most upland-like; leaf appearance, from widest, longest and most lowland-like to shortest, thinnest and most upland-like; canopy colour from bluest and most typically lowland to darkest green and most typically upland. This visual approach is akin to basic selection criteria often used by switchgrass breeders.

To assess phenotypic structure in these data, we used a DAPC ${ }^{101}$. Prior groups were determined by first transforming the phenotypic data using principal component analysis (PCA), then the first 10 principal components were used in a $k$-means algorithm to classify individuals into 3 possible groupings aiming to maximize the variation between groups. Next, DAPC was implemented on the 10 retained principal components to provide an efficient description of the ecotypic clusters using two synthetic variables, which are linear combinations of the original phenotypic variables that have the largest between-group variance and the smallest within-group variance (that is, the discriminant functions).

We classified each of the 651 tetraploid genotypes surveyed for the 16 traits at the $\mathrm{MI}$ and $\mathrm{TX}_{2}$ gardens ( 34 total features, 32 quantitative and 2 qualitative ordinal traits) to 1 of the 3 ecotypes through a low-capacity neural network with 1 hidden layer and 5 units (Supplementary Data 5). The neural network was implemented in caret $^{106}$ and was trained on seven cultivars with known ecotypes (lowland: Kanlow and Alamo; coastal: High Tide and Stuart; upland:Summer, Dacotah and Sunburst) and 78 additional genotypes that were in the same SNP-based genetic cluster (Extended Data Fig.3), collected in the same states and clustered most closely in phenotypic PCA space with the exemplar cultivars. These high-affinity exemplar genotypes are printed in Supplementary Data 5. Ecotypes for the remaining 582 genotypes that were phenotyped for the ecotype classification traits were predicted with caret ${ }^{106}$. By using traits collected at gardens representing both the northern and southern switchgrass range, we hoped to avoid local climate bias on plant phenotype and subsequent ecotype classification. Furthermore, the neural network classification approach offers one notable advantage over both DAPC and expert's qualification: because the neural network is anchored to known and published genotypes, experimentation that includes these common cultivars will be able to more effectively recapitulate our assignments.

\section{Admixture and introgression block calculation and dating}

We built a database of admixture-informative SNPs through a two-step pipeline. First, ancestry coefficients were calculated as in 'Population Genomics' from fourfold degenerate sites that had associated ancestral-state calls. The 30 samples with the least missing data and proportion of genome-wide admixture $\leq 0.001$ for each subpopulation were used to define subpopulation-specific allele frequencies. These libraries were used to find SNPs with at least one pairwise $F_{\mathrm{ST}}$ value $>0.4$, as calculated with the 'W\&C84' method in the snpRelate function snpGdsFst. Second, these global ancestry-informative sites were parsed within each subpopulation to those with minor allele frequencies $>0.05$ and missingness $<0.05$. These sites were further pruned within subpopulations first to sites with $|r|<0.9$ (10 SNPs or 1,000-bp windows), then to $|r|<0.95$ (1,000 SNPs or 10,000-bp windows) in snpRelate. This process resulted in the following SNP and library counts for each subpopulation: Atlantic, 579,468 SNPs and 284 libraries; GULF, 641,975 SNPs and 215 libraries; and Midwest, 481,563 SNPs and 196 libraries.

To test for the physical locations of admixture blocks between each pair of subpopulations, we used Ancestry_HMM ${ }^{36,107}$. This approach leverages allele frequencies in putative parental populations to determine regions of likely introgressions in a test population. For each of the three subpopulations, we sought to determine the timing, extent and current positions of admixture block introgressions. In each case, we permitted two pulses from each of the other two subpopulations. Ancestry_HMM can optimize the number of generations before present when an ancestry pulse occurred and the proportion of individuals involved in the admixture pulse. However, 8-parameter optimization with $>480,000$ sites and $>150$ libraries was not computationally feasible. Therefore, we optimized parameters using 40 randomly sampled libraries with admixture coefficients within the 0.2-0.8 quantiles of the admixture proportion distribution and SNPs only on chromosome 4 of the N subgenome. We chose this chromosome as representative of others because of a lack of obvious large high-frequency introgressions. The resulting ancestry pulse parameter optimizations were founded on an initially unadmixed population 10,000 generations before present, and two subsequent admixture pulses for each of the other two subpopulations; the optimized pulses are as follows (source-reference): Midwest - Atlantic $\left(n_{\text {generations }}=8,658\right.$ and $P_{\text {admixed }}=0.001 \% ; 67$ and $\left.0.7 \%\right)$, Gulf-Atlantic (85 and 1.1\%; 17 and 0.25\%), Atlantic-Gulf (79 and 1.9\%; 11 and $0.38 \%$ ), Midwest-Gulf (79 and $0.86 \%$; 11 and $0.14 \%$ ), Atlantic-Midwest ( 66 and $0.27 \% ; 14$ and $0.036 \%$ ), and Gulf-Midwest ( 71 and $0.15 \%$; 14 and $0.033 \%)$. These pulses were supplied to the full model with all individuals and chromosomes, along with an error probability of 0.001 , maximum number of generations before present of 10,000 and effective population size of 100,000 . Posterior ancestry probabilities were decoded into haplotype blocks and blocks were binned into clusters of similarly positioned blocks.

\section{Landscape genomics}

Geographical maps were made with publicly available layers downloaded from Natural Earth (https://www.naturalearthdata.com/). Various plotting routines rely on the $\mathrm{ff}^{108}$ and raster ${ }^{109}$ packages in the R environment for statistical computing ${ }^{110}$. Climate data were downloaded from WorldClim ${ }^{22}$ (19 bioclimatic variables, 0.5 -arcmin resolution 1960-2000) and ClimateNA ${ }^{21}$. The distribution of climate variables across collections sites was explored via dynamic clustering ${ }^{111}$ followed by partitioning around medoids clustering ${ }^{112}$ with $k=7$. The most representative climate variables were defined as those most correlated with the first eigenvector of variation within each cluster. Six of the seven clusters included WorldClim variables.

Weather data were downloaded from the NOAA portal for the most proximate weather station to each garden site that had complete daily temperature (minimum-maximum), and precipitation data from 1 September 2018 to 31 October 2019. The NOAA weather station identifiers used for each garden are as follows: IL (USC00110338), MI (USW00014815), MO (USW00003945), NE (USC00255362), OK (USW00053926), SD (USC00391076), TX (USC00414810), TX $_{2}$ (USC00410433), $\mathrm{TX}_{3}$ (USC00418862) and TX 4 (USW00003901).

Climate-phenotype associations across gardens were conducted on both raw data and imputed data. Latitude-survival associations (Fig. 2b) were accomplished on raw data with logistic regressions via glm with a binomial family in R. Imputations, which were accomplished 
in base $\mathrm{R}$ using nearest neighbours across all available phenotypes $(k=5)$, were used exclusively for tests of the rank order of gardens (Fig. 2c, d). Climate similarity-biomass associations were accomplished in mixed linear models via Imer ${ }^{92}$, comparing the full model (fixed = climate distance + intercept, random = genotype identifier) to a reduced model without the climate distance fixed effect using a likelihood ratio test.

Species distribution modelling (SDM) was used to simulate modern-day potential ranges for all ecotypes (upland, lowland and coastal) of $P$. virgatum. The final datasets used to build the SDMs comprised 277 (upland), 199 (coastal) and 121 (lowland) occurrence records. Six environmental predictors were used in our final SDM modelling $(\mathrm{BIO} 1=$ annual mean temperature, $\mathrm{BIO} 2=$ mean diurnal range, $\mathrm{BIO} 4=$ temperature seasonality, $\mathrm{BIO} 5=$ maximum temperature of warmest month, $\mathrm{BIO16}=$ precipitation of wettest quarter and $\mathrm{BIO17}=$ precipitation of driest quarter).SDMs were then generated with BIOMOD2 v.3.3 ${ }^{113}$ with seven modelling algorithms: generalized linear models, boosted regression trees, artificial neural networks, flexible discriminant analysis, random forest, classification tree analysis and multivariate adaptive regression splines. For each model, the occurrence data were coupled with 500 pseudo-absence data generated randomly within the modelled study area with equal weighting for presences and pseudo-absences ${ }^{114}$. Models were trained with $80 \%$ of the coupled occurrences and pseudo-absence data and tested with the remaining $20 \%$. Each modelling algorithm was run 100 times for a total of 700 models, which were evaluated via true skill statistics (TSS) ${ }^{115}$. TSS values ranging from 0.2 to 0.5 were considered poor, from 0.6 to 0.8 useful, and $>0.8$ good to excellent ${ }^{116}$. Unique ensemble SDMs were computed from approximately the 50 best SDMs out of 700 models for the three ecotypes on the basis of TSS threshold values (upland TSS threshold $=0.96$, lowland TSS threshold $=0.93$ and coastal TSS threshold $=0.965$ ). The final ensemble SDMs were projected onto present climate layers to visualize modern-day potential ranges (Supplementary Data 12).

We examined how the presence of Midwest introgressions in the Atlantic subpopulation were associated with the independent and joint influences of climate, geography and kinship, by implementing redundancy analysis in vegan ${ }^{117-120}$. To partition explainable variance in introgression presence attributable to climate, kinship and geography, we ran four models: one full model with introgression presence (potential introgression blocks were coded as 0 for Atlantic inheritance or 1 for Midwest introgression) explained by climate (that is, the seven representative climate variables), kinship (the first two principal components calculated from the set of putatively neutral markers) and geography (latitude and longitude), and three models for each of these three factors conditioned on the other two. The inertia (that is, variance) values from the constrained matrix of each model were compared to determine the relative importance of climate, kinship, geography and their joint effect. Furthermore, to find introgression regions strongly linked to climate and survival-corrected biomass, we extracted the loadings for the redundancy analysis axes from two additional models: (1) one predicted by only climate and (2) one predicted only by survival-corrected biomass. Both models were significant according to permutation tests ( $n=999 ; P<0.001$ for both), and all axes were approximately normally distributed. SNPs loading at the tails of each axis were more likely to indicate selection related to the predictors (that is, climate or survival-corrected biomass), so we identified all markers that were at least $2.5 \mathrm{~s}$.d. (two-tailed $P=0.012$ ) from the centre as introgressions putatively under selection ${ }^{119}$.

\section{GWAS}

Owing to the large sizes of our common garden datasets, we developed a pipeline-the switchgrassGWAS R package (https:// github.com/Alice-MacQueen/switchgrassGWAS) - to allow fast, less-memory-intensive GWAS on the diversity panel, and to analyse the extent to which SNP effects were similar or different for phenotypes measured at different sites. This package leverages bigsnpr ${ }^{121}$ to perform fast $(>300 \times$ faster than TASSEL) statistical analysis of massive SNP arrays encoded as matrices. It also incorporates current gold standards in the human genetics literature for SNP quality control, pruning and imputation, as well as population structure correction in GWAS. To test the significance of many effects in many conditions (for example, multiple sites, climate variables and so on), we used mashr ${ }^{32}$, a flexible, data-driven method that shares information on patterns of effect size and sign in any dataset for which effects can be estimated on a condition-by-condition basis for many conditions and SNPs. We determined which SNPs had evidence of significant phenotypic effects using local false sign rates, which are analogous to false discovery rates but more conservative (in that they also reflect the uncertainty in the estimation of the sign of the effect $)^{122}$. We used these values to find SNPs with $\log _{10}$-transformed Bayes factors $>2$. Here, the Bayes factor was the ratio of the likelihood of one or more significant phenotypic effects at a SNP to the likelihood that the SNP had only null effects. Following previous work ${ }^{33}$, a Bayes factor of $>10^{2}$ is considered decisive evidence in favour of the hypothesis that a SNP has one or more significant phenotypic effects.

To calculate regional heritability for climate- and fitness-associated SNPs we followed a previously described two-step method ${ }^{123}$. Variance component analysis was accomplished with ASReml (VSN International), using genomic relationship matrices calculated using the van Raden method ${ }^{124}$. Genomic relationship matrices were calculated within each subpopulation and for the full diversity panel. A kinship matrix based on all SNPs used in the univariate GWAS was calculated $(G)$, as well as a kinship matrix based on SNPs significantly associated with climate in that subpopulation ( $\log _{10}$-transformed Bayes factor $>2 ; Q_{\text {climate }}$ ) and a kinship matrix based on SNPs significantly associated with biomass or winter survival in that subpopulation ( $\log _{10}$-transformed Bayes factor $>2$, or $>1.385$ for Gulf subpopulation; $Q_{\text {fitness }}$ ). These kinship matrices were used for regional heritability mapping ${ }^{123}$ as in a previous publication $^{125}$, using mixed models of the form:

$$
\begin{gathered}
\mathbf{y}=1+Z u+Z v+e \\
\operatorname{Var}(u)=G \sigma_{u}^{2} \\
\operatorname{Var}(v)=Q \sigma_{v}^{2} \\
\operatorname{Var}(e)=I \sigma_{e}^{2}
\end{gathered}
$$

in which the vector $y$ represents the biomass values, $Z$ is the design matrix for random effects, $u$ is the whole genomic additive genetic effect, $v$ is the regional genomic additive genetic effect and $e$ is the residual. Matrix $G$ is the whole genomic relationship matrix using all SNPs for the whole genome additive effect. Matrix $Q$ is the regional genomic relationship obtained as above: one of $Q_{\text {climate }}$ or $Q_{\text {fitness. }}$. Iis the rank- $y$ identity matrix, in which $y$ is equal to the number of biomass values. Whole genomic, regional genomic and residual variances are $\sigma_{u}^{2}, \sigma_{v}^{2}$ and $\sigma_{e}^{2}$, respectively. Phenotypic variance $\left(\sigma_{\mathrm{p}}^{2}\right)$ is $\sigma_{u}^{2}+\sigma_{v}^{2}+\sigma_{e}^{2}$. Whole genomic heritability, regional heritability and total heritability are $h_{u}^{2}=\left(\sigma_{u}^{2} / \sigma_{\mathrm{p}}^{2}\right), h_{v}^{2}=\left(\sigma_{v}^{2} / \sigma_{\mathrm{p}}^{2}\right)$ and $h_{u+v}^{2}=\left(\sigma_{u}^{2}+\sigma_{v}^{2} / \sigma_{\mathrm{p}}^{2}\right)$, respectively.

These models were run for the three locations where subpopulation GWAS were conducted: Columbia, Missouri; Hickory Corners, Michigan; and Austin, Texas. This resulted in 80 models: 4 sets of populations (the full diversity panel and 3 subpopulations), 2 model types (one model with $G$ only and a $G+Q$ model), for 10 phenotypes (biomass at 3 sites and 7 environmental variables).

Variance component analyses were also used to partition variance between the K-and N-subgenomes. Only SNPs with ancestral state calls (Supplementary Data 9) were used in this analysis, resulting in 460,429 
SNPs used for each population subset. Kinship matrices based on all SNPs on a particular chromosome were calculated $\left(Q_{\text {ChrolK }}\right.$ to $Q_{\text {Chro9k }}$, and $Q_{\text {Chro1N }}$ to $Q_{\text {Chro9N }}$ ), resulting in 18 kinship matrices. These kinship matrices were used for regional heritability mapping, using mixed models of the form:

$$
\begin{gathered}
\mathbf{y}=1+Z v_{1 \mathrm{~K}}+Z v_{1 \mathrm{~N}}+Z v_{2 \mathrm{~K}}+\ldots+Z v_{9 \mathrm{~N}}+e \\
\operatorname{Var}\left(v_{i}\right)=Q_{i} \sigma_{v_{i}}^{2} \\
\operatorname{Var}(e)=I \sigma_{e}^{2}
\end{gathered}
$$

in which the vector $y$ represents the biomass values, $Z$ is the design matrix for random effects, $v_{1 \mathrm{~K}}$ (to $v_{9 \mathrm{~K}}$ ) or $v_{1 \mathrm{~N}}$ (to $v_{9 \mathrm{~N}}$ ) (collectively designated $v_{i}$ ) are the chromosome-specific genomic additive genetic effects and $e$ is the residual. Matrices $Q_{i}$ are the chromosome-specific genomic relationship matrices for the nine chromosomes of the $\mathrm{N}$ and $\mathrm{K}$ subgenomes. Chromosome-specific and residual variances are $\sigma_{v_{i}}^{2}$ and $\sigma_{e}^{2}$, respectively. Chromosome-specific heritability is $h_{v_{i}}^{2}=\left(\sigma_{v_{i}}^{2} / \sigma_{\mathrm{p}}^{2}\right)$, and subgenome-specific heritability is the sum of these variances across the nine chromosomes within each subgenome.

\section{Candidate gene exploration}

We integrated multiple data structures to rank and provide meaningful culling criteria for candidate genes within introgression intervals and physical proximity to quantitative trait loci peaks. In the case of GWAS peaks, candidate genes were defined as those loci within a 20-kb interval surrounding the mashr peak. Candidate genes for genomic introgressions must have at least partially overlapped the introgression interval. As inference of GWAS and introgressions were conducted within genetic subpopulations, all statistics reported in Supplementary Data 7 (candidate gene lists) are also subpopulation-specific, with the exception of gene co-expression analysis (which was conducted only on AP13 RNA-sequencing libraries used for annotation purposes (Supplementary Data 3)). For a given interval, we present a set of statistics. First, the physical proximity to the peak location was calculated as the midpoint of the gene to the midpoint of the interval (introgression) or GWAS peak position. Second, as the causal locus underlying GWAS peaks within a subpopulation must necessarily be variable within that subpopulation, we extracted all SNPs within and proximate to candidate gene models. These variants were annotated with SNPeff ${ }^{126}$ and the weighted sum of three main categories of variants (high, moderate and low; a description of these can be found at https://pcingola.github.io/ SnpEff/se_inputoutput/\#effect-prediction-details) for each gene were calculated as SNPeff_score $=$ high $\times 20+$ moderate $\times 5+$ low $\times 1$. Third, for each gene, we calculated the minor allele frequency of structural and presence-absence variants. Fourth, we include a vector of the identity of the WGCNA clusters for each gene. Finally, if the candidate was a homologue of flowering-time GWAS candidate genes from a previous publication ${ }^{127}$, the identity of the overlapping interval or gene is included.

\section{Reporting summary}

Further information on research design is available in the Nature Research Reporting Summary linked to this paper.

\section{Data availability}

Sequence Read Archive accession codes for all RNA and DNA sequencing libraries can be found in Supplementary Data 3 and 4, respectively. The v5 AP13 genome has been deposited at DDBJ/ENA/GenBank under the ac cession JABWAI000000000. The genome, gene and repeat annotations can also be downloaded directly from Phytozome at https:// phytozome-next.jgi.doe.gov/info/Pvirgatum_v5_1. Whenever possible, plant material will be shared upon request. Source data are provided with this paper.

\section{Code availability}

Custom pipelines for GWAS and other analyses are available from dataverse at https://doi.org/10.18738/T8/J377KE.

52. Xiao, C.-L. et al. MECAT: fast mapping, error correction, and de novo assembly for single-molecule sequencing reads. Nat. Methods 14, 1072-1074 (2017).

53. Chin, C.-S. et al. Nonhybrid, finished microbial genome assemblies from long-read SMRT sequencing data. Nat. Methods 10, 563-569 (2013)

54. Kent, W. J. BLAT-the BLAST-like alignment tool. Genome Res. 12, 656-664 (2002).

55. Li, H. \& Durbin, R. Fast and accurate short read alignment with Burrows-Wheeler transform. Bioinformatics 25, 1754-1760 (2009).

56. McKenna, A. et al. The Genome Analysis Toolkit: a MapReduce framework for analyzing next-generation DNA sequencing data. Genome Res. 20, 1297-1303 (2010).

57. Sharma, M. K. et al. Targeted switchgrass BAC library screening and sequence analysis identifies predicted biomass and stress response-related genes. Bioenerg. Res. $\mathbf{9}$, 109-122 (2016).

58. Marçais, G. \& Kingsford, C. A fast, lock-free approach for efficient parallel counting of occurrences of $k$-mers. Bioinformatics 27, 764-770 (2011).

59. Smit, A. F., Hubley, R. \& Green, P. RepeatMasker, http://www.repeatmasker.org/ (1996).

60. Ellinghaus, D., Kurtz, S. \& Willhoeft, U. LTRharvest, an efficient and flexible software for de novo detection of LTR retrotransposons. BMC Bioinformatics 9, 18 (2008).

61. Katoh, K. \& Standley, D. M. MAFFT multiple sequence alignment software version 7: improvements in performance and usability. Mol. Biol. Evol. 30, 772-780 (2013).

62. Paradis, E. \& Schliep, K. ape 5.0: an environment for modern phylogenetics and evolutionary analyses in R. Bioinformatics 35, 526-528 (2019).

63. Bennetzen, J. L. et al. Reference genome sequence of the model plant Setaria. Nat. Biotechnol. 30, 555-561 (2012).

64. Lovell, J. T. et al. The genomic landscape of molecular responses to natural drought stress in Panicum hallii. Nat. Commun. 9, 5213 (2018).

65. Wu, T. D. \& Nacu, S. Fast and SNP-tolerant detection of complex variants and splicing in short reads. Bioinformatics 26, 873-881 (2010).

66. Zuo, C. et al. Revealing the transcriptomic complexity of switchgrass by PacBio long-read sequencing. Biotechnol. Biofuels 11, 170 (2018).

67. Haas, B. J. et al. Improving the Arabidopsis genome annotation using maximal transcript alignment assemblies. Nucleic Acids Res. 31, 5654-5666 (2003).

68. Slater, G. S. C. \& Birney, E. Automated generation of heuristics for biological sequence comparison. BMC Bioinformatics 6, 31 (2005).

69. Lamesch, P. et al. The Arabidopsis Information Resource (TAIR): improved gene annotation and new tools. Nucleic Acids Res. 40, D1202-D1210 (2012).

70. Schmutz, J. et al. Genome sequence of the palaeopolyploid soybean. Nature $\mathbf{4 6 3}$, 178-183 (2010).

71. Jain, R. et al. Genome sequence of the model rice variety KitaakeX. BMC Genomics 20, 905 (2019).

72. Mamidi, S. et al. A genome resource for green millet Setaria viridis enables discovery of agronomically valuable loci. Nat. Biotechnol. 38, 1203-1210 (2020).

73. Paterson, A. H. et al. The Sorghum bicolor genome and the diversification of grasses. Nature 457, 551-556 (2009).

74. Gordon, S. P. et al. Extensive gene content variation in the Brachypodium distachyon pan-genome correlates with population structure. Nat. Commun. 8, 2184 (2017).

75. UniProt Consortium. UniProt: a worldwide hub of protein knowledge. Nucleic Acids Res. 47, D506-D515 (2019).

76. Smit, A. \& Hubley, R. RepeatModeler Open-1.0, http://www.repeatmasker.org/ (2010)

77. Bao, W., Kojima, K. K. \& Kohany, O. Repbase update, a database of repetitive elements in eukaryotic genomes. Mob. DNA 6, 11 (2015).

78. Emms, D. M. \& Kelly, S. OrthoFinder: solving fundamental biases in whole genome comparisons dramatically improves orthogroup inference accuracy. Genome Biol. 16, 157 (2015).

79. Goodstein, D. M. et al. Phytozome: a comparative platform for green plant genomics. Nucleic Acids Res. 40, D1178-D1186 (2012).

80. Schliep, K. P. phangorn: phylogenetic analysis in R. Bioinformatics 27, 592-593 (2011).

81. Subramanian, A. R., Kaufmann, M. \& Morgenstern, B. DIALIGN-TX: greedy and progressive approaches for segment-based multiple sequence alignment. Algorithms Mol. Biol. 3, 6 (2008).

82. Talavera, G. \& Castresana, J. Improvement of phylogenies after removing divergent and ambiguously aligned blocks from protein sequence alignments. Syst. Biol. 56, 564-577 (2007).

83. Guindon, S. et al. New algorithms and methods to estimate maximum-likelihood phylogenies: assessing the performance of PhyML 3.0. Syst. Biol. 59, 307-321 (2010).

84. Sanderson, M. J. r8s: inferring absolute rates of molecular evolution and divergence times in the absence of a molecular clock. Bioinformatics 19, 301-302 (2003).

85. Anders, S., Pyl, P. T. \& Huber, W. HTSeq-a Python framework to work with high-throughput sequencing data. Bioinformatics 31, 166-169 (2015).

86. Love, M. I., Huber, W. \& Anders, S. Moderated estimation of fold change and dispersion for RNA-seq data with DESeq2. Genome Biol. 15, 550 (2014).

87. Langfelder, P. \& Horvath, S. WGCNA: an R package for weighted correlation network analysis. BMC Bioinformatics 9, 559 (2008).

88. Li, H. et al. The Sequence Alignment/Map format and SAMtools. Bioinformatics 25, 2078-2079 (2009).

89. Koboldt, D. C. et al. VarScan 2: somatic mutation and copy number alteration discovery in cancer by exome sequencing. Genome Res. 22, 568-576 (2012). 
90. O'Connell, J. et al. Haplotype estimation for biobank-scale data sets. Nat. Genet. $\mathbf{4 8}$, 817-820 (2016).

91. Danecek, P. et al. The variant call format and VCFtools. Bioinformatics 27, 2156-2158 (2011).

92. Bates, D., Mächler, M., Bolker, B. \& Walker, S. Fitting linear mixed-effects models using Ime4. J. Stat. Softw. 67, https://doi.org/10.18637/jss.v067.i01 (2015).

93. Azad, A., Pavlopoulos, G. A., Ouzounis, C. A., Kyrpides, N. C. \& Buluç, A. HipMCL: a high-performance parallel implementation of the Markov clustering algorithm for large-scale networks. Nucleic Acids Res. 46, e33 (2018).

94. VanBuren, R. et al. Single-molecule sequencing of the desiccation-tolerant grass Oropetium thomaeum. Nature 527, 508-511 (2015).

95. McCormick, R. F. et al. The Sorghum bicolor reference genome: improved assembly, gene annotations, a transcriptome atlas, and signatures of genome organization. Plant J.93, 338-354 (2018).

96. Sedlazeck, F. J. et al. Accurate detection of complex structural variations using single-molecule sequencing. Nat. Methods 15, 461-468 (2018).

97. Jeffares, D. C. et al. Transient structural variations have strong effects on quantitative traits and reproductive isolation in fission yeast. Nat. Commun. 8, 14061 (2017).

98. Zheng, X. et al. A high-performance computing toolset for relatedness and principal component analysis of SNP data. Bioinformatics 28, 3326-3328 (2012).

99. Remington, D. L. et al. Structure of linkage disequilibrium and phenotypic associations in the maize genome. Proc. Natl Acad. Sci. USA 98, 11479-11484 (2001).

100. Purcell, S. et al. PLINK: a tool set for whole-genome association and population-based linkage analyses. Am. J. Hum. Genet. 81, 559-575 (2007).

101. Jombart, T., Devillard, S. \& Balloux, F. Discriminant analysis of principal components: new method for the analysis of genetically structured populations. BMC Genet. 11, 94 (2010).

102. Jombart, T. \& Ahmed, I. adegenet 1.3-1: new tools for the analysis of genome-wide SNP data. Bioinformatics 27, 3070-3071 (2011).

103. Pritchard, J. K., Stephens, M. \& Donnelly, P. Inference of population structure using multilocus genotype data. Genetics 155, 945-959 (2000).

104. Schiffels, S. \& Durbin, R. Inferring human population size and separation history from multiple genome sequences. Nat. Genet. 46, 919-925 (2014).

105. McArtor, D. B., Lubke, G. H. \& Bergeman, C. S. Extending multivariate distance matrix regression with an effect size measure and the asymptotic null distribution of the test statistic. Psychometrika 82, 1052-1077 (2017).

106. Kuhn, M. et al. Caret: Classification and Regression Training. R package version 6.0-78. https://CRAN.R-project.org/package=caret (2016).

107. Medina, P., Thornlow, B., Nielsen, R. \& Corbett-Detig, R. Estimating the timing of multiple admixture pulses during local ancestry inference. Genetics 210, 1089-1107 (2018).

108. Pebesma, E. Simple features for R: standardized support for spatial vector data. R J. 10 439 (2018).

109. Hijmans, R. J. et al. raster: Geographic Data Analysis and Modeling. R package version 3.4-5. https://CRAN.R-project.org/package=raster (2015).

110. R Core Team. R: A Language and Environment for Statistical Computing, https:// www.r-project.org/ (R Foundation for Statistical Computing, 2013).

111. Langfelder, P., Zhang, B. \& Horvath, S. dynamicTreeCut: Methods for Detection of Clusters in Hierarchical Clustering Dendrograms. R package version 1.63-1. https:// CRAN.R-project.org/package=dynamicTreeCut (2014).

112. Maechler, M. et al. Cluster: Cluster Analysis Basics and Extensions. R package version 1-56. https://CRAN.R-project.org/package=cluster (2012).

113. Thuiller, W., Georges, D., Engler, R., \& Breiner, F. biomod2: Ensemble Platform for Species Distribution Modeling. R package version 3.3-7. https://CRAN.R-project.org/ package=biomod2 (2016).

114. Barbet-Massin, M., Jiguet, F., Albert, C. H. \& Thuiller, W. Selecting pseudo-absences for species distribution models: how, where and how many? Methods Ecol. Evol. 3, 327-338 (2012).

115. Allouche, O., Tsoar, A. \& Kadmon, R. Assessing the accuracy of species distribution models: prevalence, kappa and the true skill statistic (TSS). J. Appl. Ecol. 43, 1223-1232 (2006).

116. Coetzee, B. W. T., Robertson, M. P., Erasmus, B. F. N., van Rensburg, B. J. \& Thuiller, W. Ensemble models predict Important Bird Areas in southern Africa will become less effective for conserving endemic birds under climate change. Glob. Ecol. Biogeogr. 18, 701-710 (2009).

117. Oksanen, J., Blanchet, F., Kindt, R., Legendre, P. \& Minchin, R. vegan: Community Ecology Package. R package version 2.0-10. https://CRAN.R-project.org/package=vegan (2013).

118. Gugger, P. F., Ikegami, M. \& Sork, V. L. Influence of late Quaternary climate change on present patterns of genetic variation in valley oak, Quercus lobata Née. Mol. Ecol. 22, 3598-3612 (2013).

119. Napier, J. D., de Lafontaine, G. \& Hu, F. S. Exploring genomic variation associated with drought stress in Picea mariana populations. Ecol. Evol. 10, 9271-9282 (2020).

120. Forester, B. R., Lasky, J. R., Wagner, H. H. \& Urban, D. L. Comparing methods for detecting multilocus adaptation with multivariate genotype-environment associations. Mol. Ecol. 27, 2215-2233 (2018).
121. Privé, F., Aschard, H., Ziyatdinov, A. \& Blum, M. G. B. Efficient analysis of large-scale genome-wide data with two R packages: bigstatsr and bigsnpr. Bioinformatics $\mathbf{3 4}$, 2781-2787 (2018).

122. Stephens, M. False discovery rates: a new deal. Biostat. 60, kxw041 (2016).

123. George, A. W., Visscher, P. M. \& Haley, C. S. Mapping quantitative trait loci in complex pedigrees: a two-step variance component approach. Genetics 156, 2081-2092 (2000).

124. VanRaden, P. M. et al. Reliability of genomic predictions for North American Holstein bulls. J. Dairy Sci. 92, 16-24 (2009).

125. Santantonio, N., Jannink, J.-L. \& Sorrells, M. A low resolution epistasis mapping approach to identify chromosome arm interactions in allohexaploid wheat. G3 9, 675-684 (2018).

126. Cingolani, $P$. et al. A program for annotating and predicting the effects of single nucleotide polymorphisms, SnpEff. Fly (Austin) 6, 80-92 (2012).

127. Grabowski, P. P. et al. Genome-wide associations with flowering time in switchgrass using exome-capture sequencing data. New Phytol. 213, 154-169 (2017).

128. Schnable, P. S. et al. The B73 maize genome: complexity, diversity, and dynamics. Science 326, 1112-1115 (2009).

129. Maccaferri, M. et al. Durum wheat genome highlights past domestication signatures and future improvement targets. Nat. Genet. 51, 885-895 (2019).

130. Zou, C. et al. The genome of broomcorn millet. Nat. Commun. 10, 436 (2019).

131. VanBuren, R. et al. Exceptional subgenome stability and functional divergence in the allotetraploid Ethiopian cereal teff. Nat. Commun. 11, 884 (2020).

132. Hofmeister, B. T. et al. A genome assembly and the somatic genetic and epigenetic mutation rate in a wild long-lived perennial Populus trichocarpa. Genome Biol. 21, 259 (2020).

133. Marrano, A. et al. High-quality chromosome-scale assembly of the walnut (Juglans regia L.) reference genome. Gigascience $\mathbf{9}$, giaa050 (2020).

Acknowledgements Plant collecting was conducted in collaboration with J. Randall (North Carolina Botanical Garden) through the Seeds for Success programme, A. Stottlemeyer (OSU and the USDA-NIFA Biotechnology Risk Assessment Grant Program, no. 2010-33522-21703), T. Quedensley, M. Donahue, D. Schemske and J. M. M. Reyna. We thank the Brackenridge Field laboratory, the Ladybird Johnson Wildflower Center and the Juenger laboratory for support with plant care and propagation. M. Donahue led the curation, propagation and maintenance of the diversity panel. Fieldwork was also conducted by P. Duberney, S. Reeder, K. Turner, M. Carey, T. Arredondo, N. Ryan, B. Watson, B. Battershell, N. Albert, H. Wilson, L. Simon, J. Sanley, L. Vormwald, T. Bortnem, S. Hofmann, M. Iceberg, C. Lamb and T. Vugteveen. Advice from J. G. Monroe, D. Hoover, P. Edger, J. Lasky, E. Kellogg, J. Vogel, G. Sarath and J. Tuskan helped to craft experimental designs, sequencing strategies and earlier versions of this text. R. VanBuren, P. Edger, H. Zheng, D. Ware and L. Cattivelli provided genome comparison information. We thank the HudsonAlpha Genomic Services Lab for loading Illumina X10 sequencing runs. This research was supported by the US Department of Energy Awards DESC0014156 to T.E.J., DE-SC0017883 to D.B.L. and DE-SC0010743 to K.M.D., the Great Lakes Bioenergy Research Center (Awards DESC0018409 and DE-FC02-07ER64494) and the Center for Bioenergy Innovation (Award DE-AC05-000R22725). Funding was provided by National Science Foundation PGRP Awards IOSO922457 and IOS1444533 to T.E.J. and IOS1402393 to J.T.L. The work conducted by the US Department of Energy Joint Genome Institute is supported by the Office of Science of the US Department of Energy under Contract No DE-ACO2$05 \mathrm{CH} 11231$. The work conducted by the Joint BioEnergy Institute is supported by the Office of Science of the US Department of Energy under contract no. DE-ACO2-05CH11231. The work conducted by Argonne National Laboratory is supported by the Office of Science of the US Department of Energy under contract DE-ACO2-06CH11357. J.S. thanks T. Marsh for transferring his passion for ecological science to J.S. T.E.J. thanks K. Robertson for introducing him to prairie habitats and plant diversity.

Author contributions K.D.B., P.R., M. Saha, L.E.B., M.C., K.M.D., D.B.L., D.S.R., J.G., T.E.J. and J.S. designed research. S.B., M.H., J. Jiang, T.H.P. IV, S.T., J.Z., J.M.M.-R., P.R., C.M.T., M.U., M.C., K.M.D. and D.B.L. contributed plant material and resources. J. Jenkins, C.P. and S.S. assembled and annotated the genome. J.B., A.R.B., P.A.F., F.B.F., J.D.J., D.B.L., J.L.-R., R.M., R.B.M., F.M.R. Jr, M. Saha, Y.W. and T.E.J. designed and executed field experiments. K.B., L.B., C.D., S.D., A.E., D.K., A.L., E.V.S., D.S., M. Sharma, R.S., A. Stewart, V.R.S., Y.T., J.W., X.W., M.W., Y.Y., M.Z. and R.A.W. conducted sequencing and data acquisition. J.T.L., A.H.M., S.M., J.D.N., A. Session, A. Sreedasyam, P.P.G., T.H., A.H., P.Q., C.A.S., G.A.W. and L.Z. conducted statistical and computational analyses. The manuscript was written by J.T.L., A.H.M., T.E.J. and J.S. with contributions from all authors.

Competing interests The authors declare no competing interests.

\section{Additional information}

Supplementary information The online version contains supplementary material available at https://doi.org/10.1038/s41586-020-03127-1.

Correspondence and requests for materials should be addressed to J.T.L., T.E.J. or J.S. Peer review information Nature thanks Emily Heaton, Todd Michael, lan Stavness and the other, anonymous, reviewer(s) for their contribution to the peer review of this work. Peer reviewer reports are available.

Reprints and permissions information is available at http://www.nature.com/reprints. 


\section{Article}

a Genome contiguity

\begin{aligned} & Genome attribute Size / Value \\ & Scaffold total 626 \\ & Contig total 1,090 \\ & Scaffold sequence total $1,129.9 \mathrm{Mb} \\ &$ Contig sequence total $1,125.2 \mathrm{Mb}(0.4 \%$ gap $) \\ &$ Contig N50 $5.5 \mathrm{Mb} \\ &$ Chromosome Sequence $1,093.8 \mathrm{Mb}(97.2 \%) \\ &$\hline\end{aligned}

b Genome assembly libraries

\begin{tabular}{cccc}
\hline $\begin{array}{c}\text { Sequencing } \\
\text { Platform }\end{array}$ & $\begin{array}{c}\text { Mean read/ } \\
\text { Insert Size }\end{array}$ & $\begin{array}{c}\text { n. reads } \\
(\text { M) }\end{array}$ & $\begin{array}{c}\text { Assembled } \\
\text { Coverage }(\mathrm{x})\end{array}$ \\
\hline Illumina & 500 & $1,325.3$ & 177.12 \\
PACBIO & $10,758^{*}$ & 6.983 & 83.42 \\
Total & N/A & $1,332.3$ & 260.54 \\
\hline
\end{tabular}

* Mean length of PacBio reads (bp) d Collinearity between physical position and genetic mapping position

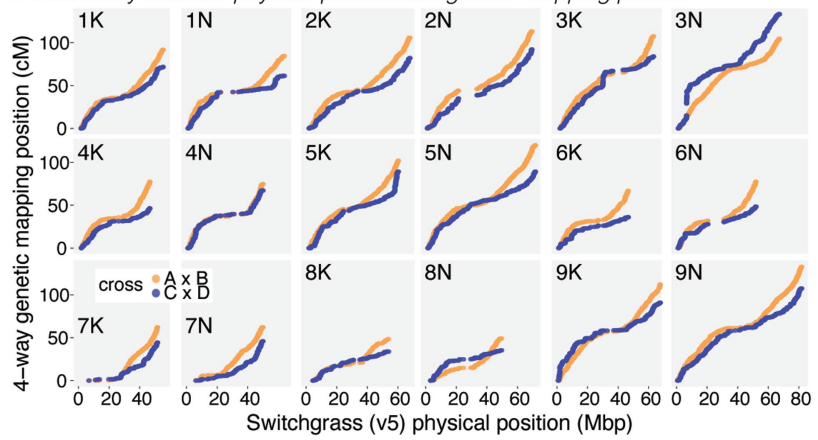

e Genome annotation statistics

Genome Attribute Size / Value

n. primary transcripts 80,278

n. alternate transcripts 49,664

Total transcripts 129,942

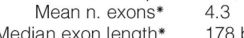

Median exon length* 178 bp

Median intron length* 139 bp

n. with RNA-seq support $\quad 69,363$

n. support ${ }^{\S}>50 \%$ of bp $\quad 67,613$

n. support ${ }^{5} 100 \%$ of bp $\quad 63,060$

* for primary transcripts only

Sgene models with $50 \%$ (100\%) of

f Heterozygosity among 732 genotypes

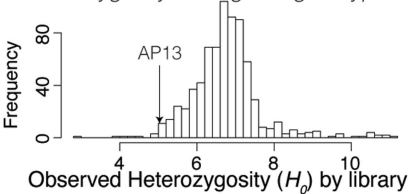

c Genome assembly statistics and metadata for a selection of outbred or polyploid plants

\begin{tabular}{|c|c|c|c|c|c|c|}
\hline Species (version) & $\begin{array}{l}\text { Genome } \\
\text { Size (Mbp) }\end{array}$ & Ploidy & Pedigree & $\begin{array}{l}\text { Heterozygous } \\
\text { bases / Mb }\end{array}$ & $\%$ repeat $^{\neq}$ & $\begin{array}{l}\text { Contiguity } \\
(\mathrm{N} 50, \mathrm{~kb})\end{array}$ \\
\hline Switchgrass (AP13 v5) & 1,125 & $4 \mathrm{x}$ & Outbred & 5,398 & $56.9 \%$ & 5,500 \\
\hline Maize (B73 refgen_v4) & 2,106 & $2 x^{*}$ & Inbred & $0^{s}$ & $60.2 \% ?$ & 1,280 \\
\hline Durum wheat (cv. Svevo) & 10,450 & $4 \mathrm{x}$ & Inbred & $0^{s}$ & $82.2 \%$ & 56 \\
\hline Broomcorn millet (Pm_0390_v0.1) & 923 & $4 \mathrm{x}$ & Inbred & $0^{s}$ & $58.2 \%$ & 369 \\
\hline Teff (Dabbi v3) & 576 & $4 \mathrm{x}$ & Inbred & 1,100 & $26.5 \%$ & 1,550 \\
\hline Poplar (P. trichocarpa v4.1) & 422 & $2 x^{*}$ & Outbred & 6,836 & $28.4 \%$ & 13,100 \\
\hline Soybean (Wm82.a4 v.1) & 978 & $2 x^{*}$ & Inbred & 59 & $46.7 \%$ & 419 \\
\hline Cotton (G. hirsutum v3.1) & 2,305 & $4 x$ & Inbred & 6 & $77.7 \%$ & 389 \\
\hline Walnut (Chander v2) & 567 & $2 x$ & Outbred & 3,800 & $58.4 \%$ & 1,100 \\
\hline Strawberry ( $x$ ananassa) & 813 & $8 x$ & Outbred & $\sim 5,000^{+}$ & $36.0 \%$ & 79 \\
\hline
\end{tabular}

"Diploid species often described as paleopolyploid; $\xi_{\text {No }}$ heterozygosity, genome was assembled as completely inbred

theterozygosity was not directly calculated, this is an estimate; ;\%of genome in repeatMasker gffl or reported; "LTR only

Extended Data Fig. 1 | Genome assembly and annotation. a-c, Genome contiguity (a) and library coverage (b) demonstrate that the $v 5$ release is a very complete genome and that it is among the best available plant reference genomes (c), compared to maize ${ }^{128}$ durum wheat ${ }^{129}$, broomcorn millet ${ }^{130}$, teff $^{131}$, poplar $^{132}$, soybean ${ }^{70}, \operatorname{cotton}^{47}$, walnut ${ }^{133}$ and strawberry ${ }^{46} . \mathbf{d}$, Complete collinearity between marker order in both crosses (number of markers $=4,701$ ) of a 4-way mapping population is evident. e, Genome annotation statistics present a gene annotation that is as complete as the assembly.f, Observed heterozygosity ranges from $<4$ to $>10 \%$ among our 732 -library resequencing panel.g, Nearly the entire single-copy genome of $P$. hallii is syntenic with both switchgrass subgenomes; pale blue polygons represent syntenic blocks between subgenomes and $P$. hallii. The one exception is a previously known

over-retained region representing the $\rho$ duplication on Chr. 03 and $08^{64}$.

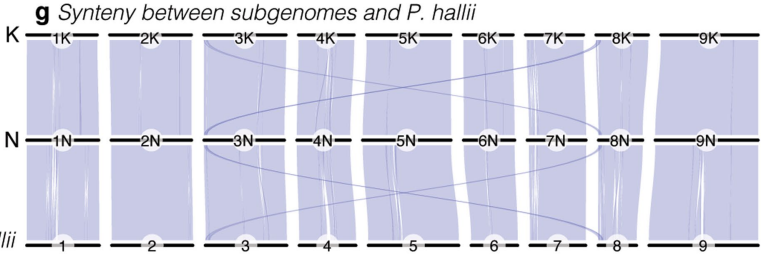

g Synteny between subgenomes and $P$. hallii

80

56

50

$$
\text { p. hat }
$$


a Summary of the 10 common gardens

\begin{tabular}{|c|c|c|c|c|c|c|}
\hline site ID & alt ID & latitude & longitude & n. genotypes & survival (\%) & biomass (mean, $\mathrm{kg}$ ) \\
\hline TX1 - & KING & 27.55 & -97.881 & 275 & 98.2 & 1628.6 \\
\hline $\mathrm{TX} 2-$ & PKLE & 30.384 & -97.729 & 579 & 100 & 1216.5 \\
\hline $\mathrm{TX} 3-$ & TMPL & 31.043 & -97.35 & 351 & 98.6 & 1387.8 \\
\hline TX4 & OVTN & 32.303 & -94.979 & 312 & 97.9 & 2151.1 \\
\hline OK & STIL & 35.991 & -97.046 & 433 & 96.8 & 1107.7 \\
\hline $\mathrm{MO}$ & CLMB & 38.897 & -92.218 & 547 & 84.3 & 1114.7 \\
\hline $\mathrm{NE}$ & LINC & 41.154 & -96.415 & 400 & 60.4 & 725.3 \\
\hline 一 & FRMI & 41.837 & -88.24 & 245 & 67.3 & 1288.2 \\
\hline $\mathrm{Ml}-$ & KBSM & 42.42 & -85.371 & 501 & 95.2 & 1088 \\
\hline$S D-$ & BRKG & 44.307 & -96.671 & 377 & 55.6 & 397.7 \\
\hline
\end{tabular}

b Climatic variation among common gardens

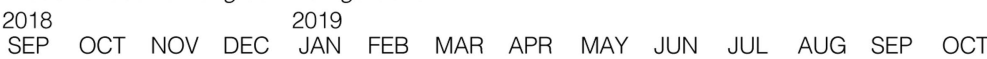

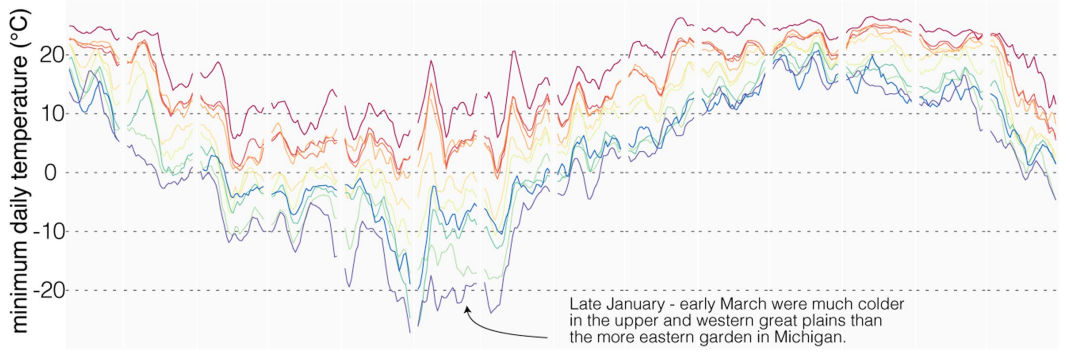

Linear days (8-Sept 2018 to 31-October 2019)

c Clustering and decription of the climate variables

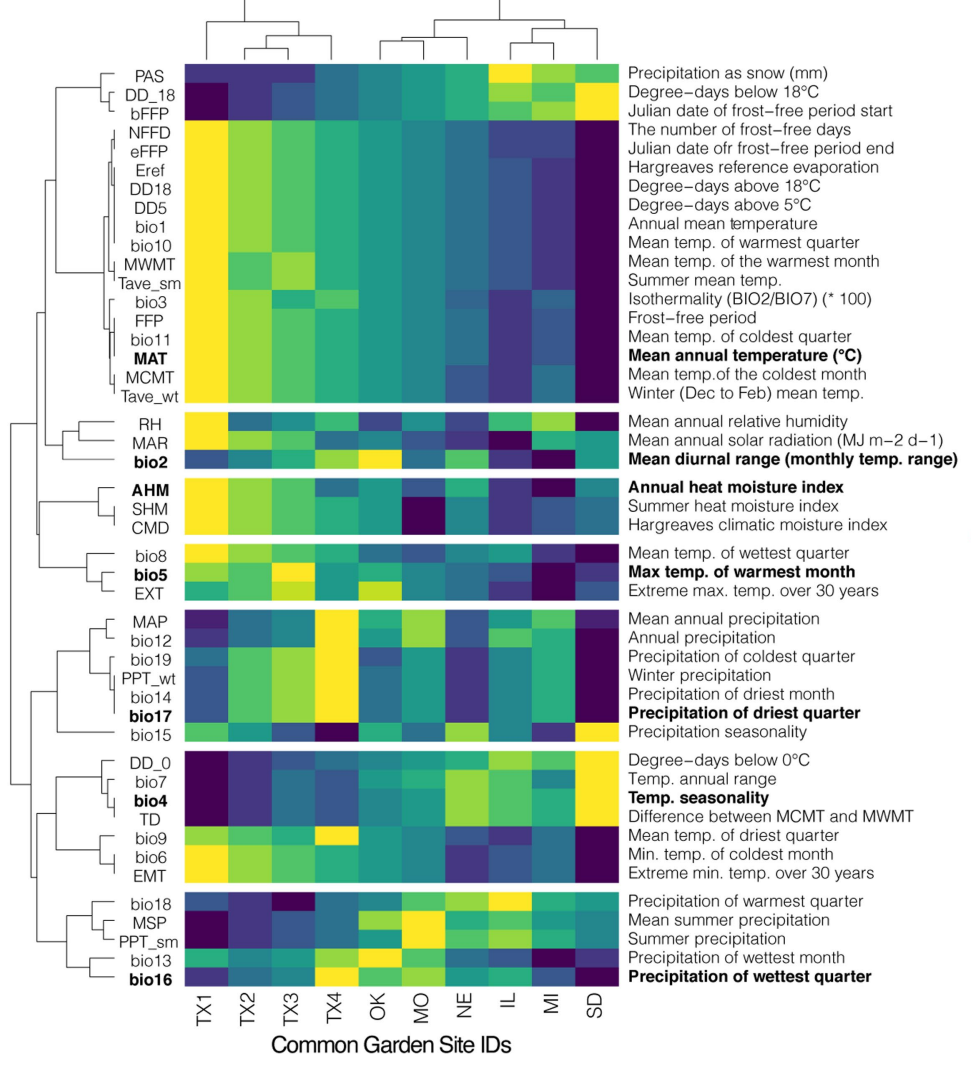

f LDA-loadings of 32 phenotypes

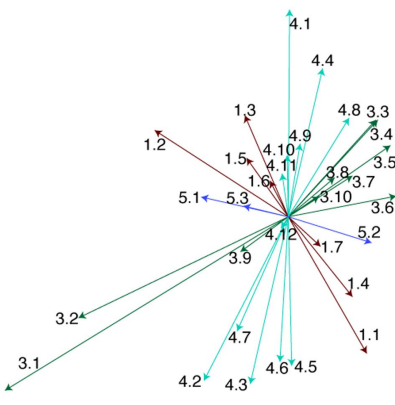

g Description of phenotypes

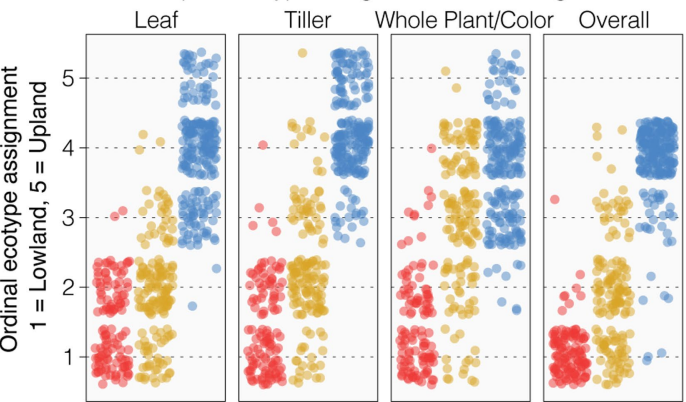

Variable ID $\mathrm{MI}$ TX2 leaf length $1.3 \quad 1.2$ leaf width $1.7 \quad 3.5$

width length (leaf) $1.5 \quad 3.9$ tiller:leaf (height) $1.4 \quad 4.8$ panicle emergence (date) $4.7 \quad 1.6$ plant size (tillers $x$ height) $3.3 \quad 3.1$ canopy height $3.2 \quad 4.3$ green-up (date) $4.1 \quad 3.7$ n. tillers (end-of-season) $4.2 \quad 3.6$ lamina:midrib (thickness) $2.1 \quad 6.1$ leaf area $3.8 \quad 4.6$ lamina thickness $3.1 \quad 5.3$

height:count (panicle) 4.54 .12

n. tillers (first cohort) 4.94 .11

height:count (leaf) $4.1 \quad 5.1$ panicle height $5.2 \quad 4.4$

h Association between biomass and climate-distance

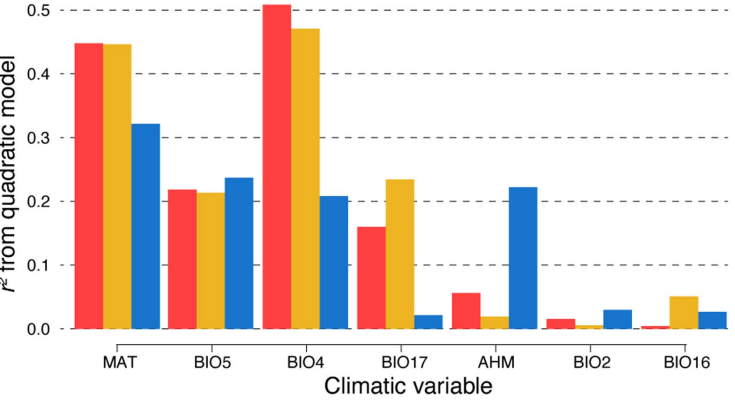

Extended Data Fig. 2 | See next page for caption. 


\section{Article}

Extended Data Fig. 2 |Phenotypic and climatic gradients among common gardens and ecotypes. a, The ten common gardens span much of the geographical distribution of, and elicit very different phenotypic responses among, our switchgrass diversity panel. For each garden, we present the georeferenced location and some basic quantitative genetic attributes of the plants grown there. b. To illustrate the climate context of winter mortality, we present a seven-day rolling mean of minimum daily temperature across the study period. Line colours match the colour key in a. c, To investigate the climatic attributes of each garden, we clustered 46 climatic variables from WorldClim (variables are named biol-1922) and ClimateNA ${ }^{21}$ using the georeferenced locations for the diversity panel; the identifiers (left) and description (right) accompany each row. These seven clusters, separated by breaks in the heat map, are represented by the seven climate variables that most closely correlated with the first principal component eigenvector of each cluster (labelled in bold). d, To investigate ecotype evolution, we probabilistically assigned each member of the diversity panel to one of three ecotypes $\left(n_{\text {upland }}=221, n_{\text {coastal }}=157, n_{\text {lowland }}=129\right)$ using a set of morphological
( $n=16$ at 2 gardens) and qualitative $(n=2)$ phenotypes; the linear discriminant functions that distinguish the ecotypes are presented here along with the eigenvectors of the two qualitative ecotype categorizations. Each point represents a single genotype grown in both $\mathrm{TX}_{2}$ and MI gardens $(n=509)$. LDA, linear discriminant analysis.e, Qualitative ecotype assessments from experts are presented for the $\mathrm{TX}_{2}$ garden in 2019. The $y$-axis scale is ordinal with five categories, but points are jittered so that the density of observations is more obvious. Points are coloured by neural network classification following d.f, Loadings for the other 16 variables (across 2 gardens) are plotted on the same scale and axes as $\mathbf{d}$. To distinguish variables, we clustered each into one of four groups, representing variation in leaf (dark green) (3), whole plant (red) (1) and combinations of these. $\mathbf{g}$, The table presents a legend for the labels in $\mathbf{f}$, in which each variable was measured in both $\mathrm{MI}$ and $\mathrm{TX}_{2}$ gardens. More detailed descriptions of the phenotypes can be found in Supplementary Data 5. h, For each of the seven climate variables, we corrected climate distance between the collection site and each common garden. The quadratic model fit $\left(r^{2}\right)$ for each variable and ecotype are presented. 
a Pairwise Fst for each combination of ecotypes and subpopulations

\begin{tabular}{ccccc}
\hline subpop1 & subpop2 & ecotype1 & ecotype2 & Fst \\
\hline ATLANTIC & ATLANTIC & Upland & Coastal & 0.033 \\
ATLANTIC & GULF & Upland & Coastal & 0.207 \\
ATLANTIC & GULF & Upland & Lowland & 0.244 \\
ATLANTIC & MIDWEST & Upland & Upland & 0.308 \\
ATLANTIC & GULF & Coastal & Coastal & 0.195 \\
ATLANTIC & GULF & Coastal & Lowland & 0.233 \\
ATLANTIC & MIDWEST & Coastal & Upland & 0.308 \\
GULF & GULF & Coastal & Lowland & 0.029 \\
GULF & MIDWEST & Coastal & Upland & 0.278 \\
GULF & MIDWEST & Lowland & Upland & 0.311 \\
ATLANTIC & GULF & & & 0.225 \\
ATLANTIC & MIDWEST & & & 0.301 \\
GULF & MIDWEST & & & 0.304 \\
OVERALL & OVERALL & & & 0.270 \\
ATLANTIC | GULF & MIDWEST & & & 0.246 \\
\hline
\end{tabular}

b Relative cross coalesence divergence

\begin{tabular}{lccccc}
\hline & mean & median & st.err & ci $(2.5 \%)$ & ci $(97.5 \%)$ \\
\hline ATLANTIC vs. GULF & 338,878 & 336,267 & 10,789 & 321,660 & 356,095 \\
GULF vs. MIDWEST & 367,310 & 369,849 & 6,819 & 350,093 & 384,528 \\
ATLANTIC vs. MIDWEST & 376,966 & 376,052 & 7,505 & 359,749 & 394,184 \\
\hline
\end{tabular}

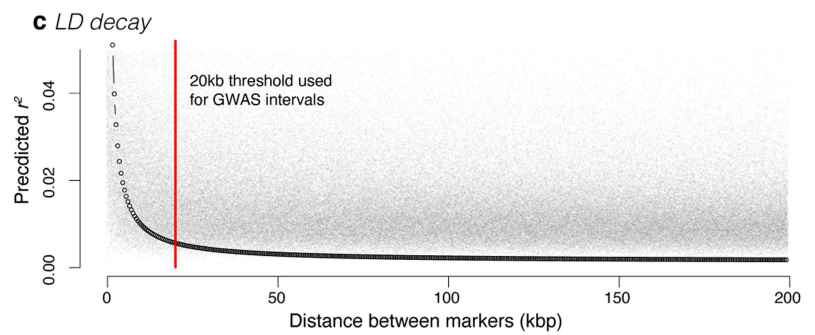

g Top 2,000 GWAS hits for each subpopulation in MI and TX2 gardens Chr $1 \mathrm{~K}$ K-Subgenome

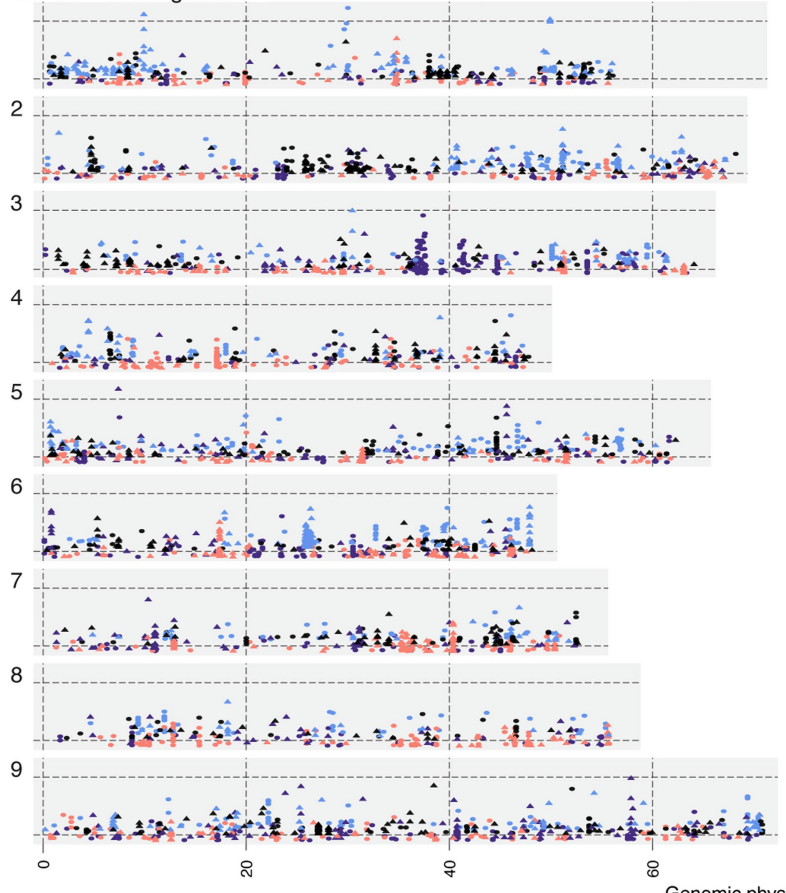

d Structural variants (SV), minor allele frequency $>10 \%$

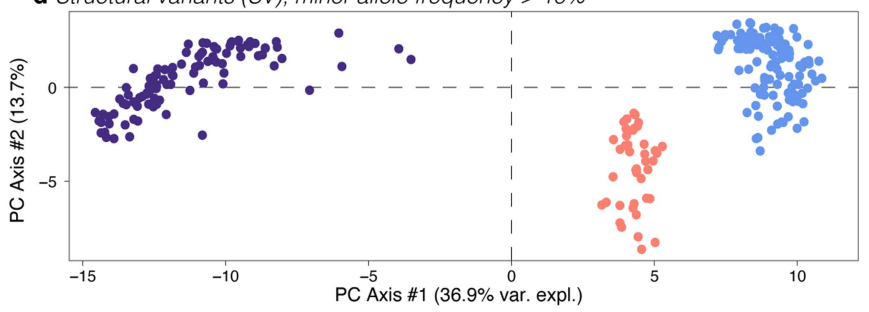

- GULF

- ATLANTIC

e Shell presence-absence variants (PAV), present in $40-60 \%$ of genotypes

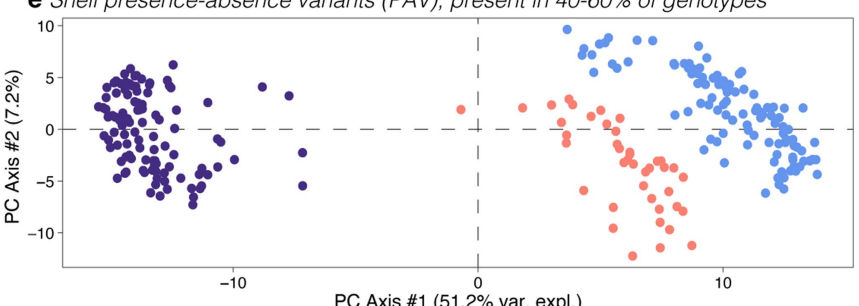

PC Axis \#1 (51.2\% var, expl.)

f Single nucleotide polymorphism (SNPS) at 4-fold degenerate sites

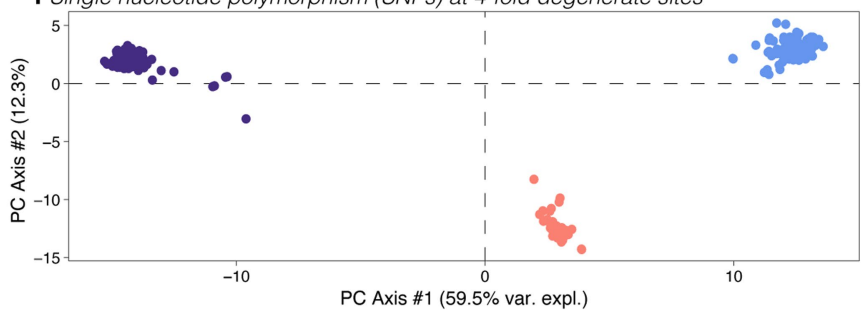

Chr $1 \mathrm{~N}$ N-Subgenome

ton

2

A

3

a

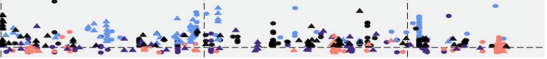

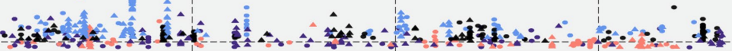

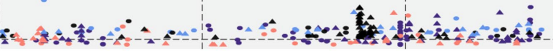

A : 1

荃:

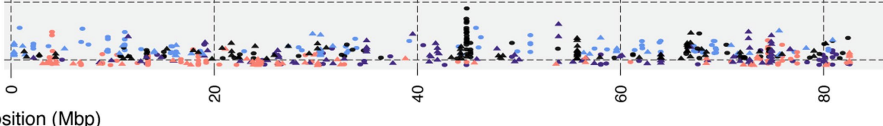

Extended Data Fig. 3 | Population and quantitative genetic divergence between and evolution within subpopulations and ecotypes. a, Pairwise $F$-statistics between each subpopulation-by-ecotype combination and across all ecotypes for each subpopulation. b, Cross coalescence (RCCR) represents an alternative method to define divergence. Here, 16 bootstraps of RCCR profiles were converted to generation time at which divergence occurred. Statistics across the bootstraps are presented.c, Linkage-disequilibrium nonlinear function of physical distance and predicted correlation coefficients among markers for the entire sample. The linear model prediction for each 500-bp interval is plotted as black open points; 2 -bp-interval mean $r^{2}$ values are the light grey points in the background. $\mathbf{d}-\mathbf{f}$, Population genetic structure is displayed as the principal coordinates from a scaled and centred distance matrix of structural variants (d), presence-absence variants (e) and SNPs (f), colour-coded by subpopulation assignments in Fig. 3. g, Positions and$\log _{10}(P$ values $)$ of the top 2,000 GWAS hits are presented for 2 gardens, the 3 subpopulations (coloured as in d-f) and an overall run (black points). 
a Subgenome bias of biomass heritability $(N-K)$ by subpopulation and garden

\begin{tabular}{rcccc}
\hline garden & GULF & ATLANTIC & MIDWEST & OVERALL \\
\hline SD & & 0.107 & 0.048 & \\
MI & 0.01 & 0.139 & 0.06 & \\
IL & 0.044 & & & 0.58 \\
NE & 0.026 & 0.091 & 0.118 & \\
MO & 0.004 & 0.021 & 0.057 & \\
OK & 0.066 & 0.013 & 0.31 & \\
TX4 & -0.088 & 0.019 & & 0.049 \\
TX3 & 0.071 & 0.016 & -0.027 & 0.037 \\
TX2 & -0.032 & 0.087 & 0.055 & 0.012 \\
TX1 & 0.002 & & & 0.054 \\
\hline
\end{tabular}

\section{c Subgenome counts across 26 tests}

\begin{tabular}{|c|c|c|c|}
\hline ID & $\mathrm{K}$ & $\mathrm{N}$ & $\mathrm{K}$ bias \\
\hline n. variable SNPS (ATLANTIC)* & $4,938,797$ & $5,661,410$ & 0.068 \\
\hline n. variable SNPs (GULF)* & $4,816,319$ & $5,503,882$ & 0.067 \\
\hline n. variable SNPS (MIDWEST) * & $4,093,574$ & $4,730,765$ & 0.072 \\
\hline n. variable SNPs (Overall)* & $4,727,783$ & $5,456,073$ & 0.072 \\
\hline n. sig. GWAS hits (climate ATLANTIC) & 2,373 & 2,139 & 0.052 \\
\hline n. sig. GWAS hits (climate GULF) & 989 & 1,128 & -0.066 \\
\hline n. sig. GWAS hits (climate MIDWEST) & 2,716 & 2,559 & 0.030 \\
\hline n. sig. GWAS hits (climate Overall) & 6,076 & 5,824 & 0.021 \\
\hline n. sig. GWAS hits (fitness ATLANTIC) & 115 & 117 & -0.009 \\
\hline GWAS hits (fitness GULF) & 5 & 4 & 0.111 \\
\hline (fitness MIDWEST) & 54 & 40 & 0.149 \\
\hline n. sig. GWAS hits (fitness Overall) & 174 & 161 & 0.039 \\
\hline n. upregulated genes (Overall) & 6,123 & 5,133 & 0.088 \\
\hline n. upregulated genes (MI) & 5,315 & 4,402 & 0.094 \\
\hline n. upregulated genes (TX2) & 5,445 & 4,477 & 0.098 \\
\hline I annotated genes & 40,957 & 38,712 & 0.028 \\
\hline n. present genes (1:1 PAV with outgroups) & 1,419 & 497 & 0.481 \\
\hline mean synonymous subst. rate $(\mathrm{ks})^{*}$ & 0.092 & 0.096 & 0.021 \\
\hline mean 4 -fold tr ratio $(4 \mathrm{dtv})^{\star}$ & 0.049 & 0.053 & 0.034 \\
\hline mean non-synon. subst. rate $(\mathrm{ka})^{*}$ & 0.029 & 0.03 & 0.022 \\
\hline total heritability (Atlantic) & 0.236 & 0.73 & -0.511 \\
\hline total heritability (Overall) & 0.079 & 0.811 & -0.823 \\
\hline ility (GULF) & 0.409 & 0.511 & -0.111 \\
\hline MIDWEST) & 0.186 & 0.807 & -0.625 \\
\hline to ATLANTIC) & $53,647,121$ & $61,626,765$ & -0.069 \\
\hline n. introgressions (MIDWEST to ATLANTIC) & 1,879 & 2,235 & -0.087 \\
\hline
\end{tabular}

*Variables where elevated values of $N$ indicated $K$ bias. These include the number of variable sites and the rate of nucleotide substitutions, since stronger purifying selection should act to reduce these.

\section{Extended Data Fig. 4 | Subgenome biases across DNA, expression and} quantitative traits. a, Difference in biomass SNP-heritability $\left(h^{2}\right)$ estimates between subgenomes for each garden-by-subpopulation combination. Garden-by-subpopulation combinations with empty cells indicate that the model did not converge. $\mathbf{b}$, Subgenome bias for all sets of genome analyses conducted here. Colours indicate the dataset used. c, Counts and ratios used to

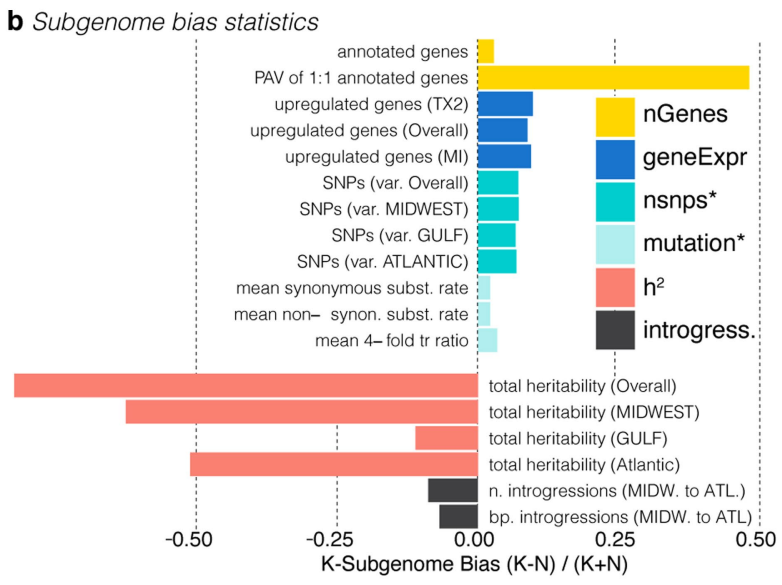

d Global mutation bias between subgenomes
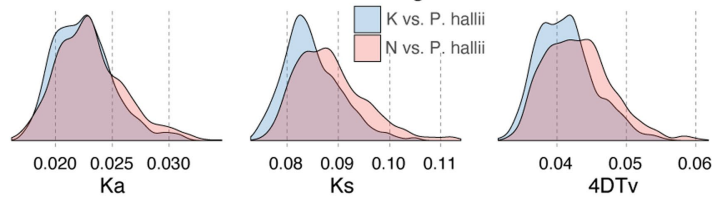

e Overall expression bias

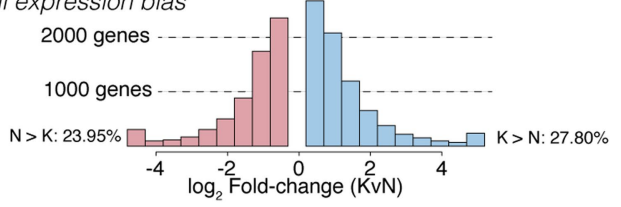

f Subfunctionalized gene expression bias

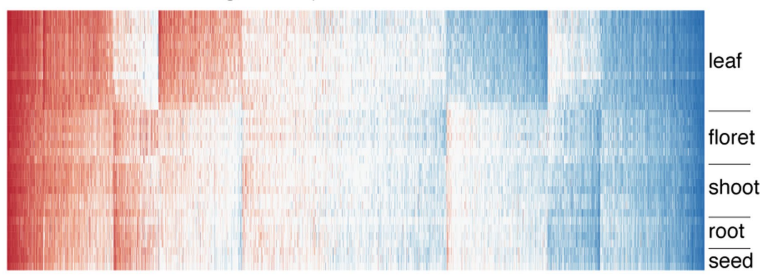

build $\mathbf{b}$, with longer descriptions of the variables. d, Density distributions of nonsynonymous $\left(K_{\mathrm{a}}\right)$, synonymous $\left(K_{\mathrm{s}}\right)$ and fourfold-degenerate transversion substation rates (4DTv) for each subgenome relative to $P$. hallii.e, Summation of the number of genes in each colour bin of $f$.f, A heat map of expression in which $\mathrm{K}>\mathrm{N}$ (blue) and $\mathrm{N}>\mathrm{K}$ (red) is shown for each tissue in the genome-annotation RNA-seq dataset. 
Extended Data Table 1| Heritability due to SNPs and background kinship

\begin{tabular}{|c|c|c|c|}
\hline subpopulation & response & $S N P-h^{2}( \pm S E)$ & polygenic background- $h^{2}( \pm S E)$ \\
\hline Atlantic & biomass_CLMB & $56.67 \pm 22.6$ & $22.64 \pm 7.3$ \\
\hline Atlantic & biomass_KBSM & $57.87 \pm 7.8$ & $7.79 \pm 6.3$ \\
\hline Atlantic & biomass_PKLE & $55.53 \pm 7.5$ & $7.49 \pm 5.3$ \\
\hline Atlantic & AHM & $38.7 \pm 8.8$ & $8.79 \pm 4.3$ \\
\hline Atlantic & bio2 & NA & NA \\
\hline Atlantic & bio4 & $82.91 \pm 17.1$ & $17.09 \pm 7.6$ \\
\hline Atlantic & bio5 & $64.31 \pm 33.7$ & $33.73 \pm 10.4$ \\
\hline Atlantic & bio16 & $80.81 \pm 19.1$ & $19.09 \pm 6.9$ \\
\hline Atlantic & bio17 & $94.13 \pm 4.9$ & $4.88 \pm 2.9$ \\
\hline Atlantic & MAT & $39.74 \pm 13.5$ & $\begin{array}{l}4.88 \pm 2.9 \\
13.53 \pm 4.6\end{array}$ \\
\hline Gulf & biomass_CLMB & $56.81 \pm 1.2$ & $1.16 \pm 16.5$ \\
\hline Gulf & biomass_KBSM & $31.66 \pm 11.2$ & $11.25 \pm 12.7$ \\
\hline Gulf & biomass PKLE & $27.84 \pm 23.3$ & $23.35 \pm 13.2$ \\
\hline Gulf & $\begin{array}{l}\text { AHM } \\
\text { AHIISS_F }\end{array}$ & $\begin{array}{l}27.44 \pm 3.3 \\
57.42 \pm 39.8\end{array}$ & $\begin{array}{l}20.35 \pm 17.2 \\
39.79 \pm 17.8\end{array}$ \\
\hline Gulf & bio2 & $51.15 \pm 47.7$ & $47.65 \pm 19.9$ \\
\hline Gulf & $\begin{array}{l}\text { bioc } \\
\text { bion }\end{array}$ & $69.03 \pm 31$ & $30.97 \pm 19.6$ \\
\hline Gulf & bio5 & $79.67 \pm 18.3$ & $18.26 \pm 18$ \\
\hline Gulf & bio16 & $38.66 \pm 61.3$ & $61.29 \pm 15$ \\
\hline Gulf & bio17 & $57.35 \pm 42.6$ & $42.57 \pm 17$ \\
\hline Gulf & MAT & $58.15 \pm 1.1$ & $1.06 \pm 11.2$ \\
\hline Midwest & biomass_CLMB & $48.12 \pm 28.9$ & $28.91+10.9$ \\
\hline Midwest & biomass_KBSM & $67.72 \pm 32.3$ & $32.28 \pm 6.4$ \\
\hline Midwest & biomass_PKLE & $61.53 \pm 29$ & $28.98 \pm 6.7$ \\
\hline Midwest & AHM & $66.67 \pm 9.4$ & $\begin{array}{l}2.90 \pm 0.1 \\
9.42 \pm 2.5\end{array}$ \\
\hline Midwest & bio2 & $96.34 \pm 2.8$ & $2.76 \pm 1.6$ \\
\hline Midwest & bio4 & $69.03 \pm 31$ & $30.97 \pm 19.6$ \\
\hline Midwest & bio5 & $98.17 \pm 1.4$ & $1.38 \pm 0.8$ \\
\hline Midwest & bio16 & $98.29 \pm 1.6$ & $1.6 \pm 1$ \\
\hline Midwest & bio17 & $97.78 \pm 2.2$ & $2.15 \pm 1.2$ \\
\hline Midwest & MAT & $72.03 \pm 2.1$ & $2.06 \pm 1.1$ \\
\hline $\begin{array}{l}\text { Full } \\
\text { Find }\end{array}$ & biomass_CLMB & $49.44 \pm 6.3$ & $6.3 \pm 5.8$ \\
\hline Full & biomass_KBSM & $44.04 \pm 0.2$ & $0.21 \pm 4.7$ \\
\hline Full & biomass PKLE & $\begin{array}{l}45.04 \pm 0.2 \\
35.93 \pm 9.1\end{array}$ & $9.08 \pm 4.5$ \\
\hline Full & AHM & $94.92 \pm 0.6$ & $0.6 \pm 10.8$ \\
\hline Full & $\begin{array}{l}\text { Alion } \\
\text { bion }\end{array}$ & $77.9 \pm 20.8$ & $20.82 \pm 12.1$ \\
\hline Full & bio4 & $50.03 \pm 50$ & $49.97 \pm 12.3$ \\
\hline Full & $\begin{array}{l}\text { bio5 } \\
\text { bios }\end{array}$ & $36.65 \pm 62.2$ & $62.24 \pm 10.5$ \\
\hline $\begin{array}{l}\text { Full } \\
\text { Full }\end{array}$ & $\begin{array}{l}\text { Dio5 } \\
\text { bio16 }\end{array}$ & $\begin{array}{l}30.65 \pm 0.2 \\
76.95 \pm 23\end{array}$ & $\begin{array}{l}62.24 \pm 1.5 \\
22.99 \pm 11.8\end{array}$ \\
\hline Full & $\begin{array}{l}\text { bio17 } \\
\text { bions }\end{array}$ & $\begin{array}{l}90.96 \pm 9 \\
9\end{array}$ & $8.95 \pm 7.5$ \\
\hline Full & MAT & $41.46 \pm 33.3$ & $33.32 \pm 9.1$ \\
\hline
\end{tabular}

Heritability of traits and climate-of-origin variation was partitioned to SNPs in GWAS hits $\left(\right.$ 'SNP- $h^{2 \prime}$ ) and background or polygenic variation. SNP-heritability and standard errors are presented for each of seven climate variables, biomass in three gardens within and across ('full') each of the subpopulations. Response variable-by-subpopulation combinations marked with 'NA' indicate that the model did not converge. 


\section{Reporting Summary}

Nature Research wishes to improve the reproducibility of the work that we publish. This form provides structure for consistency and transparency in reporting. For further information on Nature Research policies, see our Editorial Policies and the Editorial Policy Checklist.

\section{Statistics}

For all statistical analyses, confirm that the following items are present in the figure legend, table legend, main text, or Methods section.

n/a Confirmed

$\square$ The exact sample size $(n)$ for each experimental group/condition, given as a discrete number and unit of measurement

Х $\square$ A statement on whether measurements were taken from distinct samples or whether the same sample was measured repeatedly

$\triangle$ The statistical test(s) used AND whether they are one- or two-sided

Only common tests should be described solely by name; describe more complex techniques in the Methods section.

$\bigotimes$ A description of all covariates tested

$\bigotimes$ A description of any assumptions or corrections, such as tests of normality and adjustment for multiple comparisons

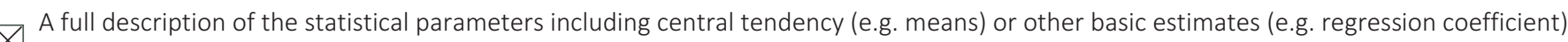

$\triangle$ AND variation (e.g. standard deviation) or associated estimates of uncertainty (e.g. confidence intervals)

$\varnothing$ For null hypothesis testing, the test statistic (e.g. $F, t, r$ ) with confidence intervals, effect sizes, degrees of freedom and $P$ value noted

Give P values as exact values whenever suitable.

$\bigotimes$ For Bayesian analysis, information on the choice of priors and Markov chain Monte Carlo settings

$\bigotimes$ For hierarchical and complex designs, identification of the appropriate level for tests and full reporting of outcomes

$\bigotimes$ Estimates of effect sizes (e.g. Cohen's $d$, Pearson's $r$ ), indicating how they were calculated

Our web collection on statistics for biologists contains articles on many of the points above.

\section{Software and code}

Policy information about availability of computer code

Data collection DNA and RNA data were collected via Illumina and PacBio internal routines. All phenotype data was collected and input manually using best practices for quantitative genetics data.

Data analysis All data analysis was conducted through programs described in the methods. The majority of which was accomplished in the R environment for statistical computing. Other programs included: ancestry_hmm, vcftools, bcftools, samtools, varscan, PLINK, SHAPEIT, bwa-mem, Picard, GATK, MSMC, GSNAP, HTSeq, Dialign-TX, Gblocks, mafft, orthofinder, Repeatmasker, RepeatModeler, PASA, EXONERATE, Jellyfish,

LTRHarvest, MECAT, BLAT, ARROW, and FlowJo. The GWAS pipeline developed here can be found on github (see code availability statement).

For manuscripts utilizing custom algorithms or software that are central to the research but not yet described in published literature, software must be made available to editors and reviewers. We strongly encourage code deposition in a community repository (e.g. GitHub). See the Nature Research guidelines for submitting code \& software for further information.

\section{Data}

Policy information about availability of data

All manuscripts must include a data availability statement. This statement should provide the following information, where applicable:

- Accession codes, unique identifiers, or web links for publicly available datasets

- A list of figures that have associated raw data

- A description of any restrictions on data availability

SRA accession codes for all RNA and DNA sequencing libraries can be found in Supplemental Data 3 and 4 respectively. The v5 AP13 genome has be deposited at DDBJ/ENA/GenBank under the accession JABWAI000000000. The genome, gene and repeat annotations can also be downloaded directly from Phytozome: https:// phytozome-next.jgi.doe.gov/info/Pvirgatum_v5_1. With the exception of map layers (Fig. 2a, Fig. 3a), which are publicly available from naturalearth.org, raw data 


\section{Field-specific reporting}

Please select the one below that is the best fit for your research. If you are not sure, read the appropriate sections before making your selection.

Life sciences

Behavioural \& social sciences

Ecological, evolutionary \& environmental sciences

For a reference copy of the document with all sections, see nature.com/documents/nr-reporting-summary-flat.pdf

\section{Ecological, evolutionary \& environmental sciences study design}

All studies must disclose on these points even when the disclosure is negative.

Study description

Research sample

Sampling strategy

Data collection

Timing and spatial scale

\section{Data exclusions}

Reproducibility

Randomization

Blinding

\section{Did the study involve field work? $\square$ res $\square$ No
Field work, collection and transport \\ Did the study involve field work? $\quad \square$ res $\quad \square$ No
Field work, collection and transport}

Field conditions

Location
We conducted quantitative analysis of phenotypes, collected in common gardens, and gene expression, collected in both common gardens and controlled conditions in the lab.

Panicum virgatum (switchgrass) plants represent the entirety of the study design. Individual genotypes were clonally replicated and phenotyped in the field. Leaf and other tissue was assayed for gene expression. Replicated measures on a single individual plant were collapsed to a breeding value and never used as a unit of replication in any analyses.

Phenotyping was always conducted in a completely randomized design within blocks (common gardens).

Field data were collected by a team of field technicians. The identities of the field techs always accompanied the measurements and care was taken to ensure that no systematic biases resulted from field technician factors.

Sample size was determined as a function of field experimental restrictions and sequencing cost. We sequenced 732 genotypes to maximize diversity within a limited budget. These plants were grown in as many sites as possible. For some sites, there was not enough space to grow all plants. In these cases, we chose plants that (a) represented the maximum genetic diversity and (b) had enough clonal replicates available.

We discuss the libraries excluded in the methods. Some libraries were excluded due to poor sequencing quality or likely contamination.

Plants were grown as clonal replicates. We opted for this approach (in lieu of full/half sib designs) to maximize repeatability: the exact same genotypes can be grown in other experiments.

At each garden, planting was completely randomized in a single block.

All field experiments were conducted using genotype identifiers that do not have an obvious connection to the location, name, etc. of each genotype. The anonymous 4- or 5-digit 'Library ID' was used for all statistical genomic analyses. It is impossible to conduct analyses blind of these identifiers, since all data is entered and output along with the IDs; however, we took care to use only these anonymous IDs and without direct reference to their biological names or context.

\begin{tabular}{ll}
\hline Field conditions & $\begin{array}{l}\text { Field conditions were ambient at } 10 \text { co } \\
\text { available, but represent far too much }\end{array}$ \\
Hocation & Here are the georeferenced coordinates \\
BRKG: 44.30680 (lat), -96.67050 (lon) \\
CLMB: 38.89690 (lat), -92.21780 (lon) \\
FRMI: 41.83671(lat), -88.23960(lon) \\
KBSM: 42.41962 (lat), -85.37127(lon) \\
KING: 27.54986(lat), -97.88101(lon) \\
LINC: 41.15430(lat), -96.41530(lon) \\
OVTN: 32.30290 (lat), -94.97940(lon) \\
PKLE: 30.38398 (lat), -97.72938(lon) \\
STIL: 35.99115 (lat), -97.04649(lon) \\
TMPL: 31.04338 (lat), -97.34950(lon)
\end{tabular}

Access \& import/export All plant collections were conducted either from established agricultural gardens under the managers permission, or from collaborators under their own collecting permits.

Disturbance

Collections of natural habitats were conducted with the utmost care by professional botanists following protocols outlined in the collection permits. Common garden field sites were always constructed in previously disturbed or agricultural lands. 


\section{Reporting for specific materials, systems and methods}

We require information from authors about some types of materials, experimental systems and methods used in many studies. Here, indicate whether each material, system or method listed is relevant to your study. If you are not sure if a list item applies to your research, read the appropriate section before selecting a response.

Materials \& experimental systems

\begin{tabular}{l|l}
\hline Involved in the study \\
$\square$ Antibodies \\
$\square$ Eukaryotic cell lines \\
$\square$
\end{tabular}

\section{Methods}

$\mathrm{n} / \mathrm{a}$ Involved in the study

Х ChIP-seq

$\square \bowtie$ Flow cytometry

\ MRI-based neuroimaging

\section{Flow Cytometry}

Plots

Confirm that:

$\bigotimes$ The axis labels state the marker and fluorochrome used (e.g. CD4-FITC).

$\bigotimes$ The axis scales are clearly visible. Include numbers along axes only for bottom left plot of group (a 'group' is an analysis of identical markers).

$\bigotimes$ All plots are contour plots with outliers or pseudocolor plots.

$\triangle$ A numerical value for number of cells or percentage (with statistics) is provided.

\section{Methodology}

Sample preparation

200-300 mg of young leaf tissue was macerated in a petri dish with a razor blade and treated for 15 minutes with $1 \mathrm{~mL}$ Cystain PI Absolute P nuclei extraction buffer (Sysmex Flow Cytometry) mixed with $1 \mu \mathrm{L} 2$-mercaptoethanol. Samples were then filtered to isolate free nuclei with a CellTrics $30 \mu \mathrm{m}$ filter (Sysmex) and treated for 20 minutes on wet ice with $2 \mathrm{~mL}$ of Cystain PI Absolute P staining buffer (Sysmex), $12 \mu \mathrm{L}$ of propidium iodide and $6 \mu \mathrm{L}$ of RNase A

Instrument

LSRFortessa SORP Flow Cytometer (BD Biosciences)

Software

FlowJo software (BD Biosciences)

Cell population abundance

NA

Gating strategy

Samples were binned into three categories based upon the average units of fluorescence per nuclei. Ploidy level of the sample was considered $4 \mathrm{X}$ if the cell population had $40-80 \mathrm{~K}$ units of fluorescence, $6 \mathrm{X}$ for $80-100 \mathrm{~K}$ units and $8 \mathrm{X}$ for $100-140 \mathrm{~K}$ units. The binning parameters were established with flow cytometry data from several P. virgatum accessions of known ploidy. T

Xick this box to confirm that a figure exemplifying the gating strategy is provided in the Supplementary Information. 